U.S. DEPARTMEAT OF COAMEACE National Tochnical Information Senice AD-A028 510

\title{
ADAPTIVE ESTIMATION AND PARAMETER IDENTIFICATION USING MULTIPLE MODEL ESTIMATION ALGORITHM
}

Massachusetts Institute of Technology

23 JUNE 1976 


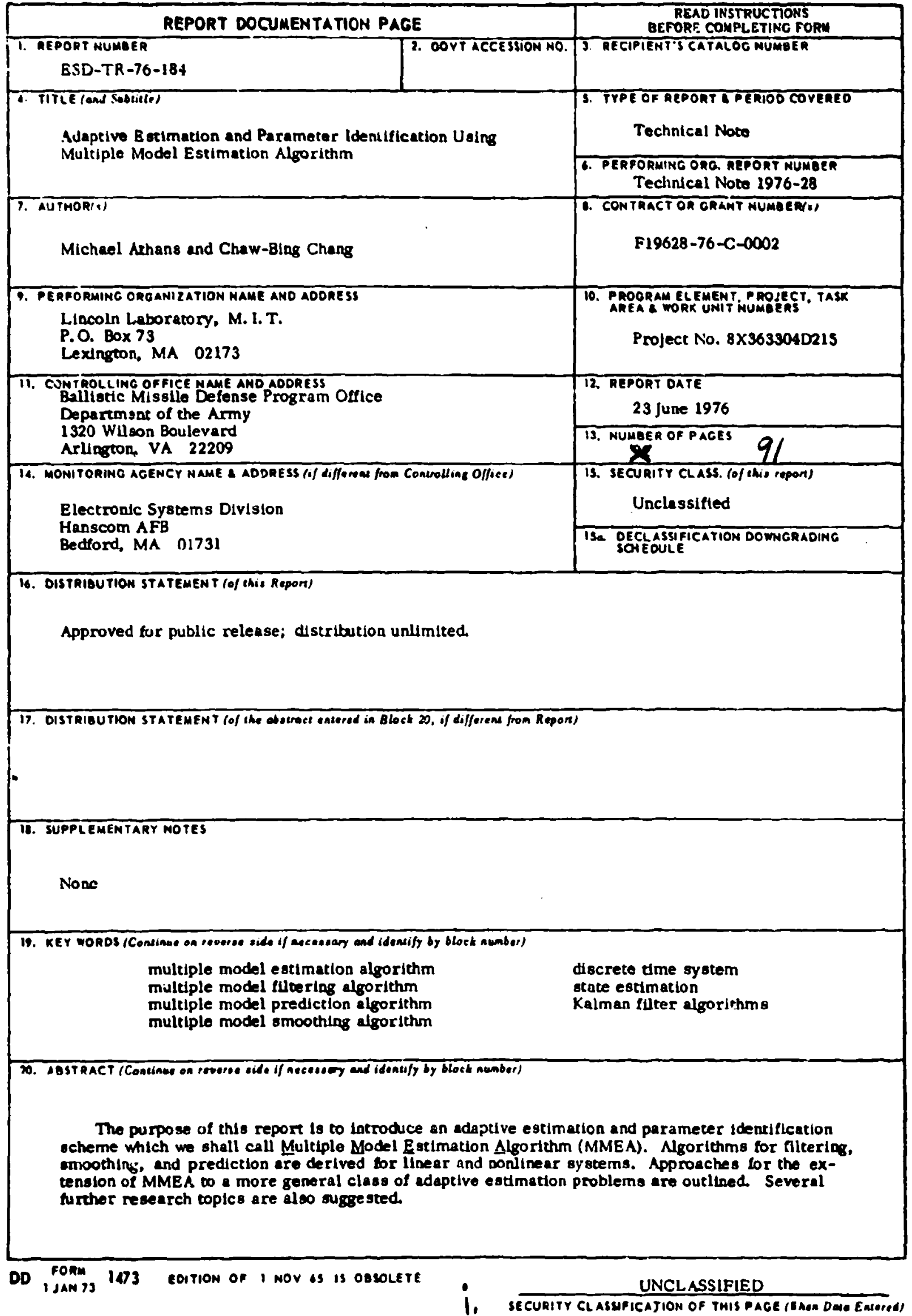




\section{MASSACHUSETTS INSTITUTE OF TECHNOLOGY}

LINCOLN LABORATORY

\section{ADAPTIVE ESTIMATION AND PARAMETER IDENTIFICATION USING MULTIPLE MODEL ESTIMATION ALGORITHM}

M. ATHANS

C. B. CHANG

Group 32

TECHNICAL NOTE 1996.28

23 JUNE 1976

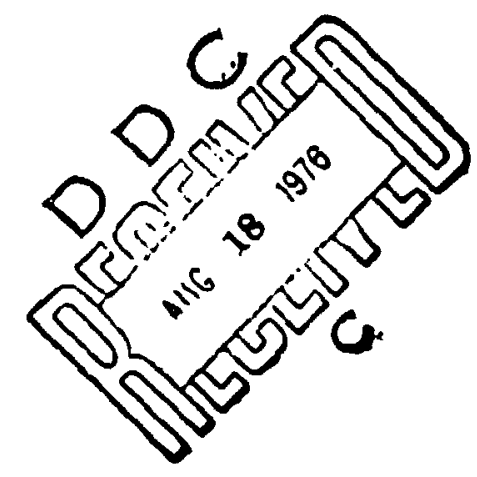

Approved for public release; distribution unlimited. 


\section{Abseract}

The purpose of this report is to introduce an adaptive estimation and parameter Identification scheme which shall call Multiple Model Eatimation Algorithm (MAEA). The MaA consists of a bank of Ralman filters with each matched to a possible parameter vector. The state estimates generated by these Kalman filters are then combined using a weighted sum with the a posteriori hypothesis probabilities as weighting factors. If one of the selected parameter vectors colncides with the true parameter vector, this algorithm gives the minimum variance state and parameter estimates. Algorlthms for filtering, smoothing, and prediction are derived for linear and nonlinesr systems. They are described in a tutorial fashion with results stated explicitly so that they can be readily used for computer implementation. Approaches for the extension of MMA to a more general class of adaptive estimation problems are outlined. Several further research topics are also suggested.

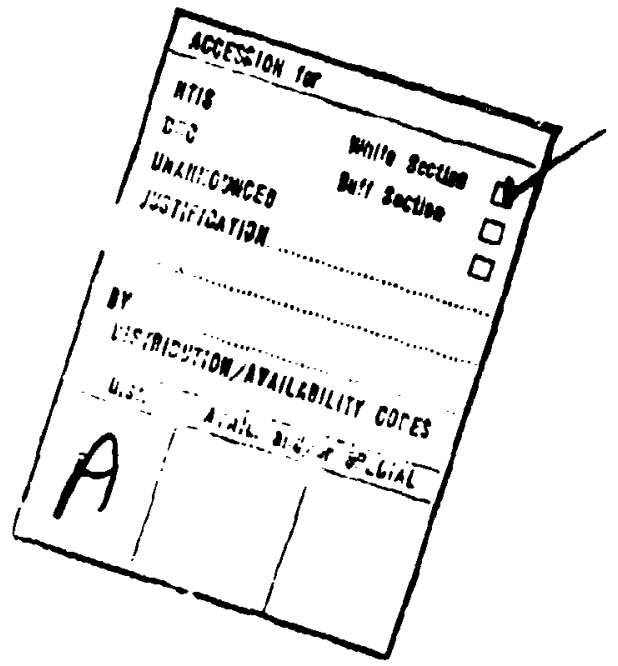




\section{Contents}

Abstract

2. INTRODUCTION

2. PROBLEM FORMULATION 5

2.1 Introduction 5

2.2 The Parameter Vector $Y$ ?

2.3 The role of $Y$ in Filtering Problems 8

2.4 Discussion 17

3. MULTIPLE MODELS FOR HYPOTHESIS TESTING AND STATE 20 ESTIMATION: FILTERING

3.1 Introduction 20

3.2 Discretization of the Parameter Space 20

3.3 Towards the MMFA; Assumptions 21

3.4 The MuAA: Formulation 22

3.5 The MMFA: Derivations 26

3.6 The MMFA: Parameter Identification 35

3.7 Discussion 37

4. MULTIPLE MODELS FOR HYPOTHESIS TESTING AND STATE 42

ESTIMATION: SMOOTHING AND PREDICTION

$\mathbf{4 . 1}$ Introduction 42

4.2 The MMSA and MMPA: Assumptions 42

4.3 The MMSA and MMPA: Derivations 43

5. MULTIPLE MODEL ESTIMATION ALGORITHM FOR NONLINEAR 47 SYSTEMS

6. EXAMPLE 51

7. SUMMARY, DISCUSSION, AND FURTHER PROBLEMS 58

7.1 Summary 58

7.2 Discussion: Extension to a Class of Time-Varying 59 Parameters and Suboptimal Approaches

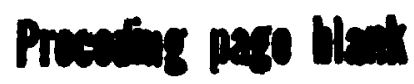




\section{InTRODOCTION}

During the past decade considerable advances have been made in the theory, algorithms, and applications of stochastic estlantion problems Involving IInear and nonlinear dynamice. The IInear Ralman filter (1) and Its diverse extensions to the nonlinear case $[2,3,4]$ are well established theoretical and algorithmic tools with extensive applications.

In most practical applications of recursive estimation theory, there are difficulties in obtaining an exact mathematical model of the physical dynamic process. The unceitain parts of the aysten are sometime represented by an unknown parameter vector. Bxamples of tr.1s kind include the ballistic coefflcient and liftIng parameters modelled in the dynamics of a reentry vehicle $[4,5,6,7,8]$. When the state estimation for this type of system has to be carried out, the varlations of these parameters and their identification play a critical role.

Many approaches have been proposed in attempting to perform state estimation together with parameter identification." One very well-known on-line ldentification method is to model the unknown parameter as a Markov process with variance related to

\footnotetext{
Teferences in this category are too many to list, one may consult the IEEE Transactions on Automatic Control (Dec. 1974), a special losue on system identiflcaiton, and reference (9) for listing of related referencea.
} 
the system atructure and the range of parameter variation. The restriction of this mathod is that lta performance is critlcally Influenced by the syatem atructure, parameter variation, and the required bias and random errors. Thls technique usually works well within a rather amall region of the state space and the varlance of the process nolie can only be determined by engineering intultion and extensive simulation study. This method however, has been able to produce excellent estimation accuracies in reentry vehicle tracking applications $[5,6,8]$.

There oxists an adaptive filtering and parameter identification method. which we shall call Multiple Model Estimation Algorithm (Mate) in this report, which has attracted considerable attentions in the academic field $[10,11,12,13,14]$. This algorithm was firat introduced by Magill (10) and later refined by Lainiotis [11]. The estimation algorithm was extended to adaptive control by w11ner [12] and Upadhyay and Lainiotis [13].

The basic concept of MIEA 18 to construct a bank of Kalman filters with each matched to a posible parameter vector value. The state estimates generated by these kalman filters are then combined using a waghted sum with the posteriori hypothesis probabilitied a weighting factors. If one of the selected parameter vectors coincides with the true parameter vector, this method gives the minimum variance estimates of both the state vector and the parameter "ector. In most physical problems, one usually has a 
good ldee of the possible values that a parameter may attain. Furthermore, the construction of the MMEA with a steady state Kalman filter bank requires only moderate computation. It therefore has attracted some attention for real-time applications $[15,16]$. The purpose of this report is to introduce the Multiple Model Bstimation Algorithm. It will be described in a tutorial fashion with results stated explicitly so that they can be readily used for computer implementation. Furthermore, the discussions on prediction and smoothing are believed to be new. Only the algorithms for discrete time system will be discussed. This is because that the modern estimation and control algorithms are mostly implemented on digital computers. Due to the fact that MMEA is theoretically more sound than the previous methods, it may be potential candidate in trajectory re-construction applications.

This report is organized as follows. In the next section, the problem of state estimation with unknown parameters is formulated. Possible solutions are discussed in a tutorial fashion. In section three, the Multiple Model Flltering Algorithm (MMFA) is derived. The extensions to prediction (MMPA) and smoothing (MMSA) are presented in section four. Discussions of the first four sections assume linear system and measurement equations. The extension to the nonlinear system and methods of algorithm realization are presented in section five. A simple second ordcr 



\section{PROBLEM FORMULATION}

\subsection{Introduction}

Consider a Iinear stochastic dynamic system whose dynam168 depend on arameter voctor $Y$. Let us write its equations In the atandard state apace representation and in the discrete time case.

\section{State Dynamics}

$$
\underline{x}(t+1)=\underline{A}(\underline{Y}) \underline{x}(t)+\underline{B}(\underline{Y}) \underline{u}(t)+\underline{\underline{L}}(\underline{Y}) \underline{\underline{\xi}}(t)
$$

\section{Measurement Equation}

$$
\underline{z}(t)=\underline{C}(\underline{\gamma}) \underline{x}(t)+\underline{\theta}(t)
$$

Next we define the different variables associated with eqs. and $(2,2)$.

The scalar $t$ is a discrete time index

$$
t=0,1,2, \ldots
$$

The state vector $\underline{x}(t) \in R_{n}$ is an $n$-dimensional vector. The input or control vector $\underline{u}(t) \varepsilon R_{m}$ is an m-dimensional vector. The plant noise vector $\xi(t) \in R_{p}$ is an $p$-dimensional vector. We assume that $\xi(t)$ represents a zero mean discrete white noise sequence with known covariance matrix $\equiv(t)$ - pxp matrix - i.e.

$$
\begin{gathered}
E\{\underline{\xi}(t)\}=\underline{0} \text { for allt } \\
\operatorname{cov}\{\underline{\xi}(t) ; \underline{\xi}(\tau)]=E\left\{\underline{\xi}(t) \underline{\xi}^{T}(\tau ;\}=\Xi(t) \delta(t, \tau)\right.
\end{gathered}
$$


where $\delta(t, \tau)$ is the Kroenecker delta

$$
\delta(t, \tau)=\left\{\begin{array}{lll}
1 & \text { iF } & t=\tau \\
0 & \text { iF } & t \neq \tau
\end{array}\right.
$$

Note that the plant noise covariance matrix $\underline{\Xi}(t)$ is symmetric and at least positive semideninite

$$
\equiv(t)=\Xi^{T}(t) \geqslant \underline{0}
$$

The measurement noise vector $\underline{\theta}(t) \in R_{I}$ is an $r$-dimensional vector. We assume that $\underline{\theta}(t)$ represents a zero mean discrete white noise sequence with known covariance matrix $\theta(t)$ - an rxr matrix - i.e.

$$
\begin{gathered}
E\{\theta(t)\}=\underline{0} \\
\operatorname{cov}[\underline{\theta}(t) ; \underline{\theta}(\tau)]=E\left\{\underline{\theta}(t) \underline{\theta}^{T}(\tau)\right\}=\underline{\theta}(t) \delta(t, \tau) \\
\underline{\theta}(t)=\underline{\theta}^{T}(t)>\underline{0}
\end{gathered}
$$

Furthermore we assume that the plant driving noise $\underline{\xi}(t)$ and the measurement noise $\underline{\theta}(\tau)$ is independent for all values of $t$ and $\tau$, i.e.,

$$
\operatorname{cov}[\underline{\xi}(t) ; \underline{\theta}(\tau)]=\underline{0} \text { for all } t, \tau
$$

The above $f i x$ the dimensions of the different matrices that appear in eqs. (2.1) and (2.2). Thus 


$$
\begin{aligned}
& \underline{A}(\gamma) \text { is an } n \times n \text { matrix } \\
& \underline{B}(\gamma) \text { is an } n \times m \text { matrix } \\
& \underline{L}(\gamma) \text { is an } n \times p \text { matrix } \\
& \underline{C}(\theta) \text { is an rxn matrix }
\end{aligned}
$$

\subsection{The Parameter Vector $\gamma$}

We have explicitly shown the dependence of the state dynamics and/or of the measurement equation upon the parameter vector $Y$. We assume that the parameter vector $Y \in R_{q}$ is a $q$-dimensional vector whose elements represent the key parameters. The elements of the parameter vector $Y$ are in general known only approximately. The degree of accuracy by which the elements of $Y$ are known are strongly dependent upon the accuracy of modelling a physical process by eqs. (2.1) and (2.2) and the experiments that have been carried out.

In general, before the initiation of any real time estimation and/or control experiments, i.e.,prior to time $t=0$, one has some idea of the nominal value of the parameter vector., denoted by $Y_{0}$, and of the degree of uncertainty (e.g.,standard deviations) associated with the nominal parameter values. For the above reasons, it is reasonable to view the parameter vector $Y$ as a random vector. All prior information about $Y$ can be captured in its prior probability density function which we shall denote by $p(\gamma)$. At the very least, our best quess about $Y$, prior to any additional real time experimentation, 
is the nominal value $y_{0}$ which we can view as the unconditional prior mean

$$
E\{Y\}=Y_{0}
$$

The degree to which we "believe" the nominal value $1_{0}$ can be communicated to the mathematics by the prior covariance matrix $I_{0}$ - a qxg matrix - of $Y, i . e$.

$$
\operatorname{cov}[Y ; Y]=E\left\{\left(Y-Y_{0}\right)\left(Y-Y_{0}\right)^{T}\right\} \Delta I_{0}
$$

It is also reasonable to assume that the uncertainty associated with the parameter vector $Y$ has nothing to do with all other uncertainties. Thus we make the assumption

$$
\begin{gathered}
x, \underline{x}(0), \underline{\xi}(t), \text { and } \underline{\theta}(\tau) \text { are independent } \\
\text { for all values of } t \text { and } \tau
\end{gathered}
$$

\subsection{The role of $Y$ in Filtering Problems}

First of all let us consider the filtering problem in the context of state estimation. To be more precise let us denote by the symbol $\mathrm{z}(t)$ the total measurements obtained from the initial time $T=0$ to the present time $t$. These measurements include both the inputs applied to the system and the actual noisy sensor measurements. Thus if we assume that the first sensor measurement is carried out at $t=1$, and that the first input is applied at $t=0$, then the data set $Z(t)$ is defined as follows 
$z(t)=\{\underline{z}(1), \underline{z}(2), \ldots, \underline{z}(t), \underline{u}(0), \underline{u}(1), \ldots, u(t-1)\}(2,15)$

In the state estimation version of the filtering problem one 18 interested in obtaining in real-time a "good" estimate of the actual value of the true state vector $\underline{x}(t)$ based upon the available data set $Z(t)$; this state estimate is commonly denoted by

$$
\underline{\hat{x}}(t / t)
$$

and the state estimation error is denoted by

$$
\underline{\tilde{x}}(t / t) \underline{\Delta} \underline{x}(t)-\underline{\hat{x}}(t / t)
$$

We can now have several cases, depending upon the relative uncertainty associated with the parameter vector $Y$.

Case 1 Parameter vector known exactly

This is an unrealistic case and corresponds to the random vector $\Upsilon$ having zero covariance

$$
\underline{I}_{0}=\underline{0}
$$

so that

$$
Y=Y_{0}
$$

Under these assumption, and the further assumption that all other random vectors, namely

$$
\underline{x}(0), \underline{\xi}(t), \underline{\theta}(\tau)
$$


are Gaussian, then the standard discrete time Kalman filter (I] generates the optimal estimate of the state in the sense that the state estimate $\underline{\hat{x}}(t / t)$ is the true conditional mean of the state

$$
\hat{\underline{x}}(t / t)=E\{\underline{x}(t) / Z(t)\}
$$

In adaition one can calculate off-line, again through the discrete time Ralman filter algorithm the true conditional covarianca $\operatorname{matrix} \sum(t / t)$

$$
\underline{I}(t / t)=\operatorname{cov}[\underline{x}(t) ; \underline{x}(t ! / Z(t)]
$$

\section{Case 2 Parameter Uncertainty relatively "small"}

In this case, we assume that the actual value of the paraneter vector $Y 18$ "very close" to its nominal value. Thus, in this case, the parameter vector covariance matrix $\Gamma_{0}$ is small.

$$
|| \Gamma_{0}||=\operatorname{small}
$$

An alternate way of characterizing this is by

$$
\| \underline{\Gamma}_{0}|| \ll|| \equiv(t)||,|| \underline{\Gamma}_{0}|| \ll|| \underline{\theta}(t)||
$$

which means that the parameter uncertainty is much smaller thar the uncertainty induced in the state by the plant noise $\underline{\xi}(t)$, and the errors introduced in the sensors by the measurement noise $\underline{\theta}(t)$. Under these circumstances, one can usually trust the robustness The discrete Ralman filter algorithm is stated in the Appendix $A$. 
of the Kalman fliter, as described in Case 1, to still generate "good" state estimates in the sense that

$$
\begin{gathered}
\underline{R}(t / t)=E \mid \underline{x}(t) / z(t)\} \\
{[(t / t)=\operatorname{cov}[\underline{x}(t), \underline{x}(t) / z(t)]}
\end{gathered}
$$

Case 3 Parameter Uncertainty Moderately low

As ||$\underline{r}_{0}||$ increases, the errors of modeliling the true values of the parameter vector $\underline{Y}$ by 1 ts nominal value $\underline{r}$ become more significant and the performance of the standard Kalman filter begins to deteriorate. In this intermediate case, and especially when the major effect of the parameter uncertainty are reflected in the state dynamics (2.1), rather than the measurement equation (2.2), there have been several cures that have been suggested.

The basic rationale is that the increased parameter incertainty in the system dynamics causes errors in the calculation of the one-step predicted estimate, $\underline{g}(t+1 / t)$. of the standard Kalman filter algorithm. These errors can only be corrected by paying more attention to the measurements, which although noisy, at1ll contain "good" information about the true state. Technically, this can be accomplished by increasing the magnitudes of the gains of the Kalman filter and, hence, the bandwidth of the Kalman filter.

One way of accomplishing this objective is to artificial- 
Iy Inereces selected elements of the plant nolle covariance antrix $\underline{\underline{g}}(t)$. This trick has been often referred to as introducIng fake white nolse. If one can get away with $1 t$, in the sense that the state estimation exrors $\underline{\underline{x}}(t / t)$ romaln accoptably sma11. then this procedure is desirable because one can still complete the (peoudo) covarlance matrix $\Sigma(t / t)$ and the Ralman fliter gaine off-line. However, this process of turning the kalman filter is more of an art than a science.

The ame philosophy of changing the magnitude of the plant noise covariance matrix $\leqq(t)$, but on an on-1ine "adaptive" mode, is by monitoring the behavior of the residuals of the kalman filter. The residual vector of time $t, \underline{\underline{r}}(t / t)$, is defined as the difference between the actual measurement at time $t, s(t)$, and the predicted measurement

$$
\underline{r}(t / t) \underline{z}(t)-\underline{c}(\underline{\underline{x}} \underline{\hat{x}}(t / t-1)
$$

In the case of no parameter uncertainty $\left(I_{0}=\underline{0}\right)$ the residuals are known to be zero-mean white and their covariance matrix, denoted by $s(t / t)$, can be calculated from $\underline{\Sigma}(t / t)$. As the parameter uncertainty increases this is reflected in the nature of the res1duals, in the sense that

(a) blases can be observed i.e.,

$$
E\{\underline{r}(t / t)\} \neq \underline{0}
$$


(b) the residuals become oorrelated in time, so that they cease to be a white noise sequence.

A variety of mothods that carry out real time tests in the rosiduals and subequently change on-line the elements of the plant nolse covariance matrix can be suggested. One of the eimplest to implement is the one suggested by Jazwinski $(2,17)$. The price that one pays in these adaptive filturing methods is increased real-time computations associated with

(a) real-time tests and computations involving the residuals

(b) subsequent transformation of the residual-derived information into changes in the covariance matrix $\underline{E}(t)$

(c) on-line calculations of the covariance equation and of the Kalman filter gain matrix

From a pragmatic point of view, these adaptive filtering algorithms change in a time-varying way the gains and the bandwidth of the Ralman filter, as modelling errors become significant and diagnosed in the residuals. If well designed, they can be effective in adjusting the bandwidth of the kalman filter.

It should be noted that there is a tradeoff associated with high-gain, high-bandwidth Kalman fllters. High-gain Ralman fliters tend to decrease mean errors rapldiy; on the other hand their high-bandwidth allows a greater amount of measursment noise 
power to pass through, and this can cause increased rus erroxe in the estimates. The successful prior timing and/or adaptive fllterIng algorithms have to take expilcitly into account these mean errora va. RHS errore tradeoffa.

\section{Care 1 Moderate Parameter Uncertainty}

As the parameter covartance matrix Io increases further, the off-line or on-line turning of the basic kalman fliter cannot be counted upon to produce good estimation accuracy. This is due to the fact that the contributions of the parameter errors to model uncertainty can no longer be taken care of as equivalent white noise.

In such cases, one has to increase the real time complexity of the algorithm 80 as to explicitly carry out some on-line parameter estimation. In other words, in order to be able to obtain reliable state estimates, one has to obtain better estimates of the parameter vector $Y$ based lipon the real time measurements. In other words, the filtering algorithm has to simultaneously generate

(a) a state vector estimate, $\hat{x}(t / t)$

(b) a parameter vector estimate, $\mathcal{P}^{(t / t)}$.

Unfortunately, even in the simplest case, the jolnt state and parameter estimation problem constitutes a nonlinear filtering problem. It is well known, [18] to [22], that the true optimal solution to a nonlinear filtering problem, in the sense 


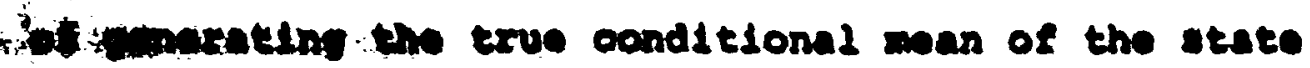

I $|\underline{x}(t) / 8(t)|$ regulres the on-line solution of a cet of nor-

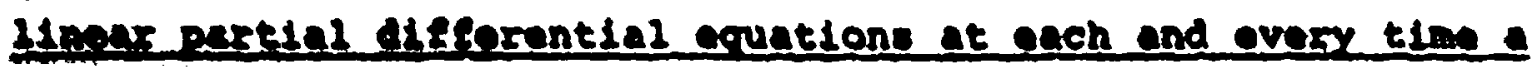

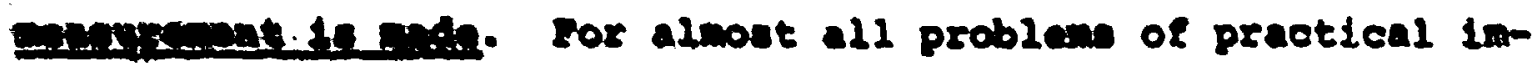
portance, the renl time computational resources force the deelgaer to use a euboptimal flltering algorithm.

The elmplest auboptimal flltering algorithin is the socalled extended xalman fliter." $A$ elightly more complex algorithin 1s the so-0olled second order (4) or gaussian $[2,23$ ) Eilter. The technique that is used to design the extended kelman eilter is that of state augmentation. Thus, in addition to 0. (2.1) which defines the dynamic stochastio evolution of the "netural" n state variables one writes another set of difference equatione of the form

$$
Y(t+1)=Y(t)
$$

In case that it is known that the parameter vector $Y$ is Indeed a conetant. If the parameter vector $I$ is known to change slowly with time, then the simplest way of modelling this is by the atochastic afference equation

$$
Y(t+1)=Y(t)+Y i t)
$$

The extended Kalman filter algorithm is stated in the Appendix A. 
where $\underline{L(t)} 1 \mathrm{~s}$ a "fake" sero mean white nolse procese with covarIenoe matrix

$$
\operatorname{cov}[\underline{\mu}(t), \underline{\mu}(\tau)]=\underline{\mu}(t) \delta(t, \tau)
$$

The covarianon matrix $\underline{M}(t)$ has to be suitably selected by the designer to reflect how rapidily and by how much one can reasonably expect the parameter $I$ to change or drift from its prior nominal value. We remark that more complex dynamic models than that shown in eq. (2.29) can be used if prior information on the "dynamics" of the parameter $Y$ is available. The extended Kalman filter algorithm that generates the state estimate $\underline{\hat{x}}(t / t)$ and the paraneter estimate $\hat{Y}(t / t)$ has much more severe computational requirements than the algorithms discussed in case 3 . These additional requirgments are due to the fact that at each measurement time one has to

(a) update an $(n+q)$ - dimensional vector, the number $(n)$ of state variables plus the number $(q)$ of the parameters

(b) propagate an $(n+q) x(n+q)$ (pseudo) covariancs matrix using the standard extended kalman filter covariance propagation formula.

(c) calculate a new $(n+q) \times r$ kalman gain matrix We remark that all the "tricks" discussed in Case 3 which involve the prior turning, or adaptive turning based on the residual be- 
havlour, can be used in this case also to shange the "fake white noise" covariance matrices $\bar{\equiv}(t)$ and $\underline{M}(t)$.

\subsection{Discussion}

The above brief semiphilosophical discussion points up some of the issues associated with the effects of uncertaln parameters upon estimation problems. One can visualize the "robustness" of the varying complexity Kalman filters described in Cases 1 to 4 as shown in Figure 2.1

The way Figure 2.1 is to be interpreted is that if the true parameter is in band 3 , then the estimators discussed in Cases 1,2 will not give satisfactory performance, while the estimators discussed in Case 3 will give good estimates. Needless to say the relative sizes or shapes of these robustness bands are next to impossible to calculate.

The point that we wish to stress, is that if the true parameter is outside the robustness band 4 , then the extended Kalman filter discussed in Case 4 cannot be trusted to generate good state estimates, even though on-line parameter estimation is accomplished. The basic reason for this is that the covariance linearizations associated with the extended Kalman filter become invalid.

For this reason we shall explain in the next section how one can attack the problem of large parameter uncertainty through hypothesis testing and subsequently suggest a suboptimal procedure 


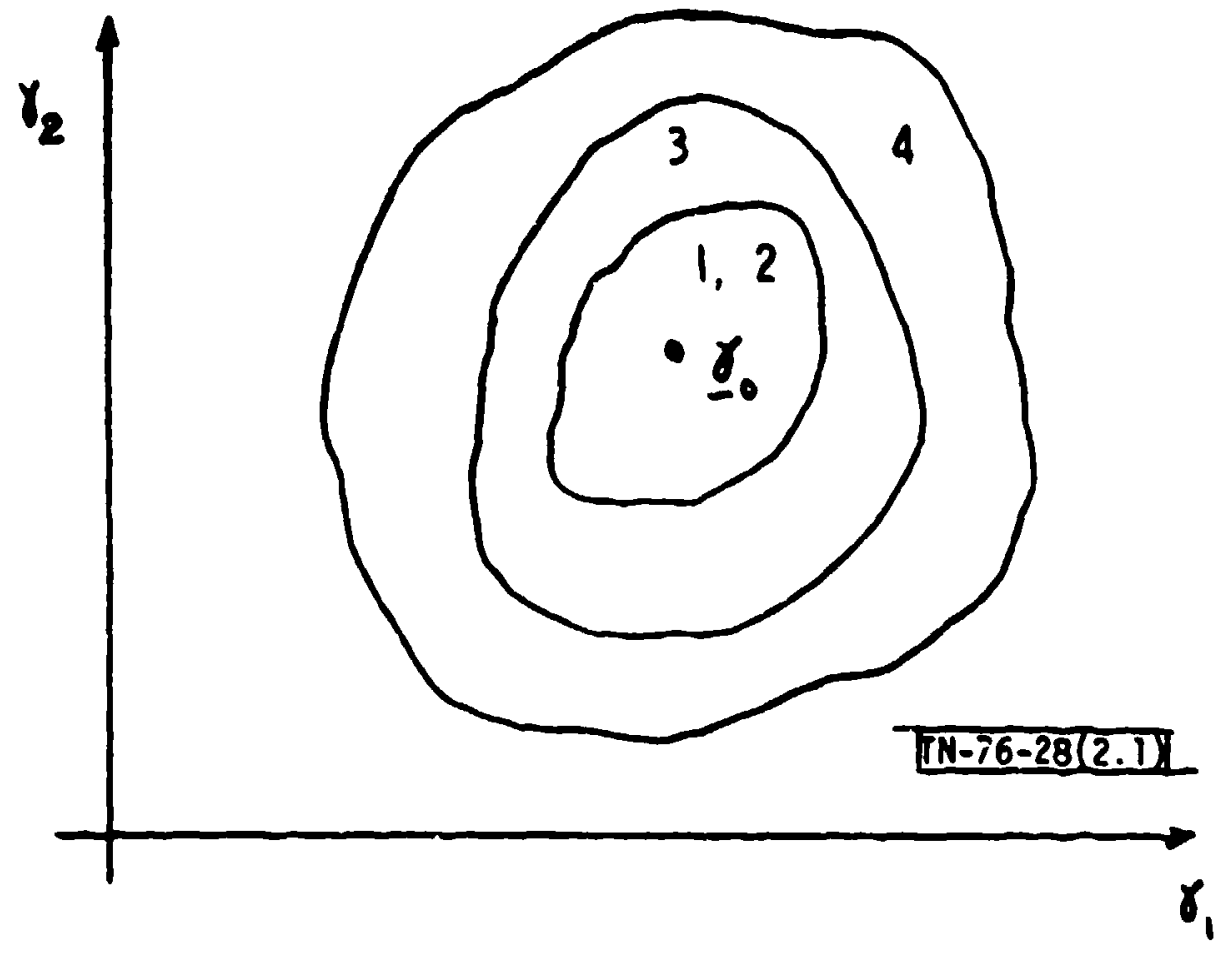

Fig. 2.1 Parameter uncertainty regions 

3. MULTIPLE MODELS FOR HYPOTHESIS TESTING AND STATE ESTIMATION: FILTFRING

\subsection{Introduction}

In the previous section we have outlined the different methods that can be employed to carry out state estimation when the system dynamics contain uncertain parameters. We have concluded that as the parameter vector variance increases one is forced to employ nonlinear filtering algorithms, e.g., the extended Kalman filter, which simultaneously estimate the parameter vector and the desired state variables. We have also remarked that even these sophisticated algorithms will break down as the parameter uncertainty increases.

In this section we present the next most obvious level of complexity to take into account the effect of uncertain parameters. The first and simplest case is to subdivide the parameter space into regions and see what happens to the state estimation algorithm when such a discretization of the parameter space is carried out.

\subsection{Discretization of the Parameter Space}

As we have remarked in section 2.2 , the parameter vector $Y$ is a q-dimensional vector. In most physical problems, one has some prior idea of the physical ranges of the elements of the parameter vector $Y$. This engineerinf, knowledge can be translated into a subset $\Omega_{\gamma}$ of $R_{q}$; the physica? significance of $\Omega_{\gamma}$ is that it represents all reasonable values that the parameter vector $Y$ can 
attain.

The next step is to select a finite set of parameter values denoted by

$$
r_{1}, r_{2}, \ldots, r_{N}
$$

These parameter vectors are scattered in the region $\Omega_{Y}$.

\subsection{Towards the MFA, Assumptions}

Let 48 suppose that the parameter vector $Y$. which appears in the state space description of the stochastic dynamic system (2.1) - (2.2) does indeed coincide with one of the $Y_{i}$ defined above. However, prior to making any measurements we do not know the "true index" i.

Needless to say, the above assumption is not true in any real life situation, in the sense that the true parameter vector $Y$ will be "near," but not identical to, one of the $Y_{1}^{\prime} s$. Once more, we shall postpone discussion of this lssue for the time being.

Under the assumption that indeed $Y$ coincides with one of the $Y_{1}^{\prime} s$ we can ask two questions:

1. What type of an algorithm can be used in order to generate

a. the true conditional mean of the state, and

b. the true conditional covariance matrix of the state

given a set of past measurements. We remark that this constitutes the standard estimation or filtering question. 
2. What type of an algorithm can be used to identify the true parameter $Y_{i}$ given a set of past measurements. We remark that this constitutes an identification question.

One may argue that in many applications one may not be interested in the identification question, but only in the state estimation problem. " Nonetheless, it turns out that one cannot answer the questions independently, but one must obtain the answer to both questions simultaneously.

We shall next formulate the problem in a mathematically precise way, and then summarize the solution algorithm.

\subsection{The MMF: Formulation}

For each value of $Y$, denoted by $Y_{i}$, let us redefine the matrices in section 2 as follows

$$
\begin{gathered}
A\left(\underline{Y}_{i}\right) \stackrel{\Delta \Delta_{i}}{=}, \underline{B}\left(Y_{i}\right) \underline{\Delta B}_{i}, \underline{L}\left(Y_{i}\right) \underline{\Delta L}_{i} \\
\underline{C}\left(Y_{i}\right) \underline{\Delta C}_{i} \quad ; i=1,2, \ldots, N
\end{gathered}
$$

We remark that the matrices $\underline{A}_{i}, \underline{B}_{i}, \underline{L}_{i}, \underline{C}_{i}$ can be time-varying; their time dependence is not explicitly shown.

\footnotetext{
In the context of tracking RV's, if one tracks a ballistic RV, and the baliistic coefficient is viewed as the uncertain parameter, then one is usually interested in both state estimation for good tracking, and parameter estimation for discrimination. A similar situation exists for maneuvering re-entry vehicles; in the MaRV case one is interested in estimating parameters that are characteristic of the magnitude and direction of the maneuver accelerations.
} 
Using the above notation, one has a class of $N$ distinct linear stochastic dynamic systems described by

State Dynamice

$\underline{x}(t+1)=\underline{A}_{1} \underline{x}(t)+\underline{B}_{1} \underline{u}(t)+\underline{I}_{1} \underline{\xi}(t) \quad ; \quad i=1,2, \ldots, N$

Measurement Equation

$\underline{z}(t)=\underline{c}_{1} \underline{x}(t)+\underline{\theta}(t) \quad ; \quad i=1,2, \ldots, N$

The characteristics of the Gaussian plant noise $\xi_{(}(t)$ are still given by eqs. (2.4) - (2.7), while the characteristics of the Gaussian measurement noise $\underline{\theta}(t)$ are still given by eqs. $(2.8)-(2.11)$.

In addition to the plant noise, measurement noise, and initial state uncertainty, we must specify the parameter vector uncertainty. Under our assumptions, the random vector $Y$ can attain a set of discrete values $Y_{1}, Y_{2}, \ldots, Y_{N}$. In view of this, $I$ is a discrete random vector.

We can model this fact by a set of hypotheses. Let $\mathrm{H}$ be a scalar random variable ( a hypothesis variable) and let

$$
\mathrm{H}_{1}, \mathrm{H}_{2}, \ldots, \mathrm{H}_{\mathrm{N}}
$$

denote a set of events.

The interpretation that we attach to the event

$$
H=H_{j} \quad \text { is } \quad Y=Y_{j}
$$


and we can think of this phenomenon as that "nature" has selected the f-th linear oyetem, defined by eqs. (3.3) and (3.4) and has placed it inside a black box.

Before we obtain any data from the system in the blork box, we have to have some idea of the prior probability of which system is in the black box, or equivalently, the probalility that $Y=Y_{1}$ for each 1 .

Let $\underline{P}_{1}(0)$ denote the prior probability that the 1 -th aystem is in the "black box." Thus

$$
P_{1}(0) \triangle \operatorname{FrOb}\left(H=H_{1}\right)=\operatorname{Prob}\left(Y=Y_{1}\right)
$$

with

$$
P_{i}(0) \geq 0, \quad \sum_{i=1}^{N} P_{i}(0)=1 .
$$

Thus, the probability density function, $F(f)$, of the random variable H is

$$
P(H)=\sum_{i=1}^{N} P_{i}(O) \delta\left(H-H_{i}\right)
$$

where $\delta(\cdot)$ is the Dirac delta function.

Remark: The numerical values of the prior probabilities $P_{f}(0)$ reflect to the mathematics our best guess on which models are more likely to be in the black box prior to their generating any data. If initially, i.e., at time $t=0$, any one of the models is equally likely, then we would select the $P_{1}(0)$ by 
and make a set of nolse meaurements

$$
\underline{\varepsilon(1)}, \underline{\varepsilon}(2), \ldots, \underline{z}(t)
$$

Erom the gretem in the black box. As we have done in section 2 we let $s(t)$ danote the set of all past measurements

$$
\underline{n}(t)=\{\underline{u}(0), \underline{u}(1), \ldots \underline{\underline{u}}(t-1), \underline{\underline{z}}(1), \ldots, \underline{\underline{z}(t)}\}
$$

-Defline the probabilities

$$
\begin{aligned}
P_{1}(t) & \Delta \operatorname{Prob}\left(H=H_{1} / Z(t)\right) \\
& =\operatorname{Prob}\left(y=Y_{1} / Z(t)\right)
\end{aligned}
$$

to be the probability, given the measurement set $8(t)$, that the 1-th hypotheels (1.e., the 1-th model) is the correct one. clearly

$$
\begin{aligned}
& P_{1}(t) \geq 0 \\
& \sum_{1=1}^{N} P_{1}(t)=1
\end{aligned}
$$


Olven all of the above Information and notation, we can liet all the Information that we would like to obtain, as well on the required algorithme to compute the variables of interest.

1. The conditionel mean of the state

$$
\underline{\hat{x}}(t / t) \Delta E\{\underline{x}(t) / Z(t)\}
$$

2. The conditional state covariance matrix

$$
\underline{\Sigma}(t / t) \underline{\Delta} \operatorname{cov}[\underline{x}(t) ; \underline{x}(t) / z(t)]
$$

3. The dynamic evolution of the posterior probabilities $\underline{P}_{1}(t)$; ideally we would like a recursive - telation, 1.e., $P_{1}(t+1)$ can be computed from the $P_{j}(t)$.

Remark: The conditional mean and the covarlance can be computed once $p(x(t) / z(t))$, the true conditional density furiction of the state of the system in the "black box" has been obtained.

\subsection{The Mapa: Derivations}

We shall abtain recursive relationships of the general conditional density functions at time $t+1$ given at time $t$.

We start by evaluating the conditional probability density function

$$
p(\underline{x}(t+1) / z(t+1))
$$

Use of the marginal density yields

$$
p(\underline{x}(t+1) / Z(t+1))=\int p(\underline{x}(t+1), H / Z(t+1)) d H
$$




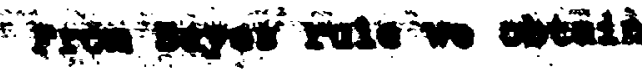

$P(x(t+1), \theta / z(t+1))=p(\underline{x}(t+1) / A, 8(t+1)) P(B / z(t+1))$

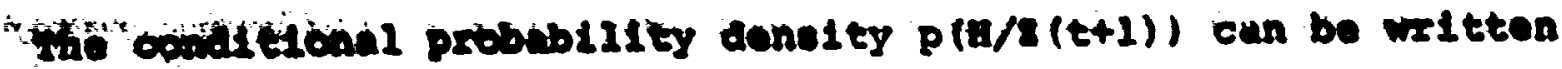
uning the motation of eq. (3.13) as

$$
p(H / z(t+1))=\sum_{1=1}^{B} P_{1}(t+1) \delta\left(H-H_{1}\right)
$$

subst1tute eqs. (3.20) and (3.21) Into oq. (3.19), and Integrate to obtaln

$$
p(\underline{x}(t+1) / z(t+1))=\sum_{1=1}^{N} P_{1}(t+1) p\left(\underline{x}(t+1) / q_{1}, z(t+1)\right)
$$

Revarkz we know that the conditional densities $P\left(x(t+1) / H_{1}, Z(t+1)\right)$ can be generated by a bank of $N$ Kalman filters where each ralman Eliter 1s "matched" to a dietinct model, 1.e.,1-th hypothoels.

It 1s important to realize from basic xalman flltering theory that the following relationship is true for each conditional probability density

$$
p\left(\underline{x}(t+1) / B_{1}, 8(t+1)\right)=\frac{p\left(\underline{\underline{z}}(t+1) / B_{1}, \underline{x}(t+1)\right) p\left(\underline{x}(t+1) / B_{1}, 8(t)\right)}{p\left(\underline{\underline{z}}(t+1) / B_{1}, 8(t)\right)}
$$

and that

$$
P\left(\underline{X}(t+1) / H_{1}, z(t)\right)=\int P\left(\underline{x}(t+1) / H_{1}, \underline{x}(t)\right) P\left(\underline{x}(t) / H_{1}, z(t)\right) \underline{d x}(t)
$$




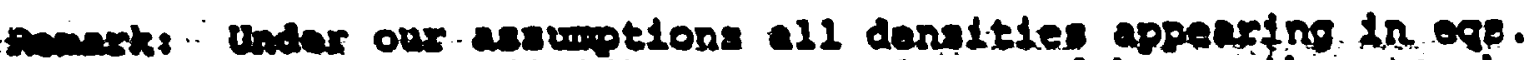
$(3.23)$ and $(3.24)$ are causelen, and honos thry oan be cheracterlsed by their moun and covarlanos matelx.

The banio ldea is to conetruct i bank of $N$ Ralang E11t-

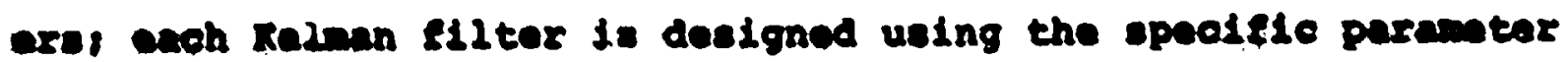
matrioes $\underline{A}_{1}, \underline{Q}_{1}, \underline{I}_{1}, \underline{C}_{1}, \underline{E}, \underline{\theta}$, and $\underline{E}_{0}$ (the Initial state covar1ance antr(x). Bach Kalman filter in the bank 18 driven by the eane Input sequence, $\underline{u}(t)$, applied to the gyetem in the "black box," and by the actual measurement sequence, $z(t)$, generated by the syetem in the "black box."

Let $\hat{x}_{1}(t / t)$ denote the state estimate generated by the 1-th Kalman f1lter. Hore precisely, $\hat{\underline{x}}_{1}(t / t)$ is deflned by.

$\left.\hat{\underline{x}}_{1}(t / t) \Delta E \mid \underline{x}(t) / H_{1}, z(t)\right\}=\int \underline{x}(t) p\left(\underline{x}(t) / H_{1}, z(t)\right) \underline{d x}(t)$

Let $\Sigma_{1}(t / t)$ denote the conditional covariance matrix aseociated with the 1-th Kalman filter. More precisely

$$
\begin{aligned}
& \underline{\varepsilon}_{1}(t / t) \Delta \operatorname{cov}\left[\underline{x}(t) ; \underline{x}(t) / H_{1}, q(t)\right] \\
& =E\left\{\left(\underline{x}(t)-\underline{\hat{x}}_{1}(t / t)\right)\left(\underline{x}(t)-\underline{\underline{x}}_{1}(t / t)\right)^{T} / H_{1}, z(t)\right\} \\
& =\int\left(\underline{x}(t)-\underline{\hat{x}}_{1}(t / t)\left(\underline{x}(t)-\underline{\hat{x}}_{1}(t / t)\right)^{T} \cdot p\left(\underline{x}(t) / H_{1}, z(t)\right) \underline{d x}(t)(3.26)\right.
\end{aligned}
$$

Remark: All the $\underline{I}_{1}(t / t), 1=1,2, \ldots . N$ are precomputable.

In essence, from each Ralman fllter mean $\hat{x}_{i}(t / t)$ and covarlance matrix $\underline{\Sigma}_{1}(t / t)$ we can construct the Gaussian density function $p\left(\underline{x}(t) / \mathbf{H}_{1}, \mathbf{z}(t)\right)$. 
The next problem is to generate an overall evtimate of the state, $\hat{\underline{x}}(t / t)$, according to $9 q .(3.16)$ of the system in the "black box." In addition, it is helpful to generate the true error covarlance matrix, $\underline{E}(t / t)$, according to eq. (3.17), so that we have an ldea of how accurate the estimate $\underline{\hat{x}}(t / t)$ of the true syatem state $\underline{x}(t)$ actually is.

We demonstrate below how the overall estimate $\underline{x}(t / t)$ can be generated once

a. The individual Kalman filter estimates $\hat{\underline{x}}_{1}(t / t)$
are avaliable, and

b. The true conditional probabilities $P_{1}(t)$ defined by eq. (3.13) are available.

From eq. (3.22) we have

$$
\begin{aligned}
& \underline{p}(\underline{x}(t) / Z(t))=\sum_{i=1}^{N} \underline{p}_{i}(t) \underline{p}\left(\underline{x}(t) / H_{1}, z(t)\right) \\
& \underline{\hat{x}}(t / t)=E\{\underline{x}(t) / Z(t)\}=\int \underline{x}(t) p(\underline{x}(t) / Z(t)) \underline{d x}(t) \\
&=\sum_{i=1}^{N} P_{i}(t) \int \underline{x}(t) \underline{p}\left(\underline{x}(t) / H_{1}, z(t)\right) \underline{d x}(t) \\
&=\sum_{i=1}^{N} P_{1}(t) \underline{\hat{x}}_{1}(t / t)
\end{aligned}
$$

Thus, the overall state estimate is the probabilistically weighted average, by the posterior (hypotheses) probabilities $P_{1}(t)$, of the state estimate generated by each one of the $N$ Kalman filters. To derive the true conditional covariance matrix $\underline{\Sigma}(t / t)$ 
m prooeed as gollows:

$$
\begin{aligned}
& \underline{E}(t / t) \Delta \operatorname{cov}[\underline{x}(t) i \underline{x}(t) / 8(t)] \\
& \text { - } \boldsymbol{I}\left\{\left(\underline{x}(t)-\underline{\hat{x}}(t / t)(\underline{x}(t)-\underline{\hat{x}}(t / t))^{T} / 8(t)\right\}\right. \\
& =\int(\underline{x}(t)-\underline{\hat{x}}(t / t))(\underline{x}(t)-\underline{\hat{x}}(t / t))^{T} p(\underline{x}(t) / 2(t)) \underline{d x}(t) \\
& \text { - } \sum_{1=1}^{\mu} P_{1}(t) f(\underline{x}(t)-\underline{\hat{x}}(t / t))(\underline{x}(t)-\underline{\hat{x}}(t / t))^{T} \text {. } \\
& \text { - } p\left(\underline{x}(t) / H_{1}, z(t)\right) \underline{d x}(t)
\end{aligned}
$$

After some algebra we obtain

$$
\begin{aligned}
\underline{\Sigma}(t / t)= & \sum_{1=1}^{N} P_{1}(t)\left[\underline{\Sigma}_{1}(t / t)+\left(\underline{\hat{x}}_{i}(t / t)-\underline{\hat{x}}(t / t)\right) \cdot\right. \\
& \left.\cdot\left(\underline{\hat{x}}_{1}(t / t)-\underline{\hat{x}}(t / t)\right)^{T}\right]
\end{aligned}
$$

Note that $\underline{\Sigma}(t)$ cannot be precomputed because it contains the real time estimates $\hat{\hat{x}}_{1}(t / t)$ generated by the kalman filters in addition to the posterior probabilities $P_{i}(t)$ which as we shall see require real time measurements. The only remaining problem is to calculate dynamic evolution of the porbabilities $P_{i}(t)$

$$
\begin{aligned}
P_{1}(t) & =\operatorname{PrCb}\left[H=H_{i} / Z(t)\right] \\
& =\operatorname{Prob}\left[Y=Y_{i} / Z(t)\right]
\end{aligned}
$$

We will relate each $P_{1}(t+1)$ to the $P_{1}(t)$ and other quantities that can be found from Kalman filters. The interesting 
aspact of this caloulation lo that a truly recureive relationanip oar be obteined relating quantities only at successive measurament tima, $t$ and $t+1$, with relatively amall computational burenen.

Towards this goal we proceed as followe. Consider the conditional denaity

$$
p(H / Z(t+1))=\sum_{i=1}^{N} P_{1}(t+1) \delta\left(H-H_{1}\right)
$$

Use of Bayes rule yields

$$
\begin{aligned}
P(H / Z(t+1)) & =P(H / z(t+1), Z(t)) \\
& =\frac{P(H, z(t+1) / Z(t))}{P(\underline{z}(t+1) / Z(t))} \\
& =\frac{P(z(t+1) / H, Z(t)) p(H / Z(t))}{P(\underline{z}(t+1) / z(t))}
\end{aligned}
$$

But

$$
P(H / Z(t))=\sum_{i=1}^{N} P_{1}(t) \delta\left(H-H_{1}\right)
$$

Note that according to our notation $z(t+1)=\{z(t), \underline{z}(t+1)\}$

Equations (3.32) to $(3.34)$ yield

$$
P_{i}(t+1)=\frac{P\left(\underline{z}(t+1) / H_{i}, Z(t)\right)}{P(\underline{z}(t+1) / Z(t))} P_{i}(t)
$$

The density $p\left(\underline{z}(t+1) / H_{i}, z(t)\right)$ is Gaussian and can be 
caloulated from the 1-th Kalman elltor

$$
p\left(\underline{\underline{z}}(t+1) / \mathrm{H}_{1}, 8(t)\right) \sim N\left(\underline{C}_{1}(t+1) \underline{\underline{\underline{x}}}_{1}(t+1 / t) \cdot \underline{\underline{s}}_{1}(t+1)\right)
$$

where

$$
\underline{s}_{1}(t+1)=\underline{c}_{1}(t+1) \underline{\Sigma}_{1}(t+1 / t) \underline{c}_{1}^{T}(t+1)+\underline{\theta}(t+1)
$$

Note that the quantity $\underline{C}_{1}(t+1) \underline{\hat{x}}_{1}(t+1 / t)$ is the predicted measurement at time $t+1$ generated by the 1 -th Kalman filter.

The matrix $\underline{s}(t+1)$ is the residual covariance matrix assoclated with the 1-th Kalman filter. Note that the residual covariance matrices $\underline{s}_{1}(t+1)$ can be calculated off-line for each Ralman filter.

It remains to calculate the density $p(z(t+1) / 2(t))$ in eq. (3.35). Use of the marginel density leads to

$$
\begin{aligned}
& p(\underline{z}(t+1) / Z(t))=\int p(\underline{z}(t+1), H / Z(t)) d H \\
& =\int p(\underline{z}(t+1) / H, Z(t)) p(H / Z(t)) d H \\
& =\int p(\underline{z}(t+1) / H, Z(t)) \sum_{j=1}^{N} P_{j}(t) \delta\left(H-H_{j}\right) d H \\
& =\sum_{j=1}^{N} P_{j}(t) p\left(\underline{z}(t+1) / H_{j}, Z(t)\right)
\end{aligned}
$$

Remark: Once more all the densities $p\left(z(t+1) / H_{1}, z(t)\right)$ are avallable from the bank of Kalman filters; See eqs. (3.36)

\footnotetext{
The notation $N(\underline{a}, \underline{A})$ denotes a Gaussian density with mean $\underline{a}$ and covarlance $A$.
} 
and (3.37). 8ubstituting a. (3.38) into a. (3.35) ylelde the desired result that the dynamic evolution of the probabilitles $\underline{P}_{1}(t)$ is given by

$$
P_{1}(t+1)=\frac{p\left(\underline{z}(t+1) / B_{1}, 8(t)\right)}{\sum_{j=1}^{N} P_{j}(t) p\left(\underline{z}(t+1) / A_{j}, z(t)\right)}-p_{1}(t)
$$

where if we recall that

$$
r=\operatorname{dim} \Sigma(t)=\text { number of measurements }
$$

then

$$
\begin{gathered}
p\left(\underline{z}(t+1) / \mathrm{u}_{j}, z(t)\right)=[2 \pi]^{-\frac{r}{2}}\left[\operatorname{det} \underline{s}_{j}(t+1)\right]^{-\frac{1}{2}} . \\
\cdot \exp \left\{-\frac{1}{2}\left(\underline{z}(t+1)-\underline{c}_{j}(t+1) \underline{\hat{x}}_{j}(t+1 / t)\right)^{T} s_{j}^{-1}(t+1)\right. \\
\left.\cdot\left(\underline{z}(t+1)-\underline{c}_{j}(t+1) \underline{\hat{x}}_{j}(t+1 / t)\right)\right\}
\end{gathered}
$$

with

$$
\underline{s}_{j}(t+1)=\underline{c}_{j}(t+1) \underline{\tau}_{j}(t+1 / t) \underline{c}_{j}^{T}(t+1)+\underline{\theta}(t+1)
$$

The relation (3.39) becomes more transparent if we introduce a somewhat simpler notation.

Let us define the residual (an r-dimensional vector) vector generated by each Kalman filter by

$$
\underline{\underline{r}}_{1}(t+1) \Delta \underline{z}(t+1)-\underline{c}_{i}(t+1) \underline{\hat{x}}_{i}(t+1 / t)
$$


1.0., the difference between the actual measurement and the predicted measurement.

Then from each Kalman filter we can obtain the scalar quantity in seal time

$$
w_{i}(t+1) \Delta \underline{r}_{i}(t+1)^{T} \underline{s}_{i}^{-1}(t+1) \underline{\underline{r}}_{i}(t+1)
$$

Also, let $\beta_{1}(t+1)$ denute the scalar precomputable quantity

$$
\beta_{i}(t+1) \triangleq[2 \pi]^{-\frac{T}{2}}\left[\operatorname{det} \underline{s}_{i}(t+1)\right]^{-\frac{1}{2}}
$$

Using the above notation, the conditional density (3.41) can be written as

$$
P\left(\underline{z}(t+1) / H_{j}, Z(t)\right)=B_{1}(t+1) \exp \left\{-\frac{1}{2} W_{i}(t+1)\right\}
$$

From egs. (3.46) and (3.39) we can now write the dynamic evolution of the probability density function as

$$
P_{i}(t+1)=\frac{B_{i}(t+1) \exp \left\{-\frac{1}{2} w_{i}(t+1)\right\}}{\sum_{j=-1}^{N} B_{j}(t+1) \exp \left\{-\frac{1}{2} w_{j}(t+1)\right\} P_{j}(t)} P_{i}(t)
$$

The above formula illustrates that all measurements up to time $t$, $z(t)$, are captured in the posterior probabilities

$$
P_{1}(t), P_{2}(t), \ldots, P_{N}(t)
$$

The new measurement at time $t+1, \underline{z}(t+1)$, influence all 
N residual vectors associated with the bank of Kalman filters according to eq. $(3.43)$ and generate scalars $w_{i}(t+1), i=1,2, \ldots, N$. This then can be used to update the probabilities

$$
P_{1}(t+1), P_{2}(t+1), \ldots, P_{N}(t+1)
$$

according to eq. (3.47). Thus, this represents a true recursive solution to the problem of probability updates.

A block diagram illustrating the MMFA is shown in figure 3.1 .

\subsection{The MFA: Parameter Identification}

In the previous subsection, we have described the basic idea of the Multiple Model Filtering Algorithm. In addition, we have derived algorithms for MMFA realizalion. In this subsection, we will show that the MMFA for parameter identification can be obtained in a straightforward manner. The minimum variance estimate of the unknown parameter $I$ is the conditional mean $1 . e .$,

$$
\hat{Y}(t)=\int \underline{Y}(\underline{Y} / Z(t)) d \underline{Y}=E[\underline{Y} / Z(t)\}
$$

Recalling the fact that the events $H=H_{i}$ and $Y=Y_{i}$ are equivalent, we can rewrite eqn. $(3.21)$ as

$$
p(\underline{Y} / Z(t))=\sum_{i=1}^{N} P_{i}(1):\left(\underline{Y}-\underline{Y}_{i}\right)
$$

where $\underline{P}_{i}(t)$ is interpreted as the probe ojlity that $Y=Y_{i}$ is true 


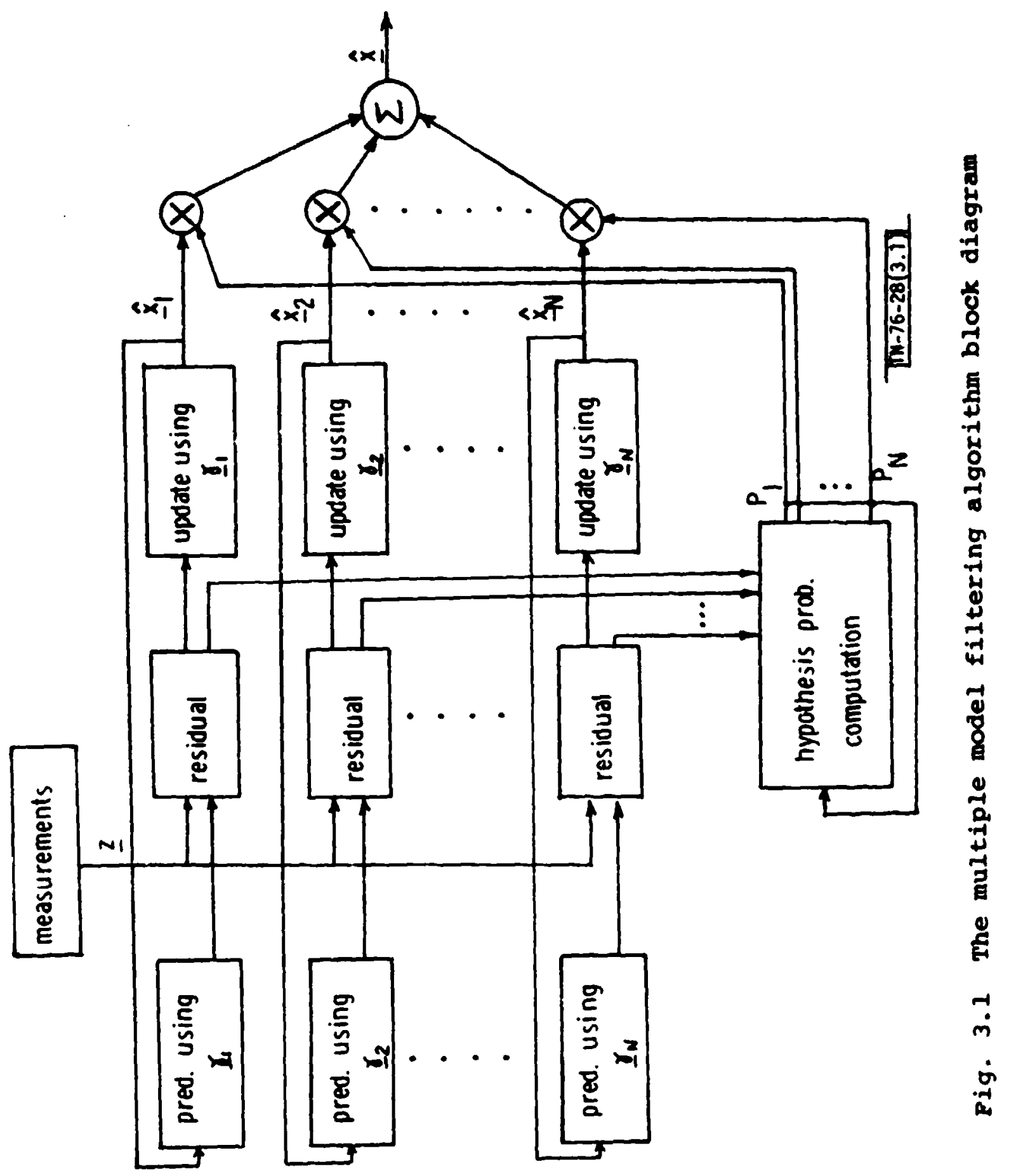


based upon all the data, $z(t)$. Using (3.51) in (3.50) yields

$$
\hat{Y}(t)=\sum_{i=1}^{N} Y_{1} P_{i}(t)
$$

The covariance of $\hat{Y}$ can be obtained similarly. Assuming that $\hat{y}$ is unbiased, then

$$
\begin{aligned}
\underline{\underline{\Sigma}}_{\hat{Y}}(t) & =\operatorname{cov}(\hat{Y}(t)) \\
& =\int(Y-\hat{Y}(t))(\underline{Y}-\hat{Y}(t)) T_{P}(Y / Z(t)) d Y \\
& =\sum_{I=1}^{M} P_{i}(t)\left[\left(\underline{Y}_{I}-\hat{Y}(t)\right)\left(Y_{i}-\hat{Y}(t)\right)^{T}\right)
\end{aligned}
$$

\subsection{Discussion}

We now discuss the asymptotic properties of this algorithm from a heuristic point of view. If the system is subject to some sort of persistent excitation, then one would expect that the residuals of the Kalman filter associated with the correct model, say the 1-th one will be "small", while the residuals of the mismatched filters $(j \neq i, j=1,2, \ldots, N)$ will be "large". Thus, if $i$ indexes the correct model we would expect

$$
w_{i}(t) \ll w_{j}(t) \quad \text { for all } j \neq i
$$

If such a condition persists over several measurements equation (3.47) shows that the "correct" probability $P_{i}(t)$ will increase while the "mismatched model" probabilities will decrease. To see this one can rewrite $(3.47)$ as follows, 


$$
\begin{aligned}
P_{1}(t+1)-P_{1}(t) & =\left[\sum_{j=1}^{N} B_{j}(t+1) \exp \left\{-\frac{1}{2} N_{j}(t+1)\right\} P_{j}(t)\right]^{-1} . \\
P_{1}(t) & {\left[\left(1-P_{1}(t) / B_{1}(t+1) \exp \left\{-\frac{1}{2} N_{1}(t+1)\right\}\right.\right.} \\
& \left.-\sum_{j N_{1}} P_{j}(t) B_{j}(t+1) \exp \left\{\frac{1}{2} w_{f}(t+1)\right\}\right]
\end{aligned}
$$

Under our assumptions

$$
\begin{aligned}
& \exp \left\{-\frac{1}{2} w_{1}(t)\right\}=1 \\
& \exp \left\{-\frac{1}{2} w_{j}(t)\right\}=0
\end{aligned}
$$

Hence the correct probability will grow according to

$$
P_{1}(t+1)-P_{1}(t)=\frac{P_{1}(t)\left[1-P_{1}(t)\right] B_{1}(t+1)}{\sum_{j=1}^{N} P_{j}(t) B_{j}(t+1) \exp \left\{-\frac{1}{2} N_{j}(t+1)\right\}}>0(3.56)
$$

which demonstrates that as $P_{1}(t)+1$, the rate of growth slows down.

On the other hand, for the incorrect model, indexed by jFl, the same assumptions yield

$$
P_{j}(t+1)-P_{j}(t)=\frac{-P_{f}(t) B_{1}(t) B_{1}(t+1)}{\sum_{j=1}^{N} P_{j}(t) B_{j}(t+1) \exp \left\{-\frac{1}{2} W_{j}(t+1)\right\}}<0
$$

so that the probabilities decreased. 
The same conclusions hold if we rewrite (3.17) in the

form

$$
\begin{gathered}
P_{1}(t+1)-P_{1}(t)-\left[\sum_{j=1}^{M} P_{j}(t) B_{j}(t+1) \exp \left\{-\frac{1}{2} w_{j}(t+1)\right\}\right]^{-1} \\
\cdot P_{1}(t)\left[\sum _ { j = 1 } P _ { j } ( t ) \left(B_{1}(t+1) \exp \left\{-\frac{1}{2} w_{j}(t+1)\right\}\right.\right. \\
\left.\left.-B_{j}(t+1) \exp \left\{-\frac{1}{2} w_{j}(t+1)\right\}\right)\right]
\end{gathered}
$$

The above discussion points out that this "Identification" scheme 18 crucially dependent upon the regularity of the residual behavlor between the "matched" and "mis-matched" Ralman filters.

As pointed out in reference [16], the dynamic evolution of the residuals may not follow the above regularity assumptions. This may be caused by errors in the selection of the noise statistics or using a steady state kalman filter design, among others. To be specific, suppose that for a prolonged sequence of measurements the Kalman filter residuals turn out to be such that

$$
w_{1}(t)=w_{2}(t)=\ldots \approx w_{N}(t)
$$

Then

$$
\exp \left\{-\frac{1}{2} w_{1}(t)\right\}=a \text { for all } 1
$$

Under this condition and using (3.58), we can see that 


$$
P_{1}(t+1)-P_{1}(t)=\frac{P_{1}(t) \sum_{j \neq 1} P_{j}(t) \cdot\left(B_{1}(t+1)-B_{j}(t+1)\right)}{\sum_{j=1} P_{j}(t) \beta_{j}(t+1)}(3.60)
$$

suppose that one of the $B_{1}{ }^{\prime}$, , say the $B_{k}$, 1s dominant, 1.e..

$$
B_{k}>B_{1} \text { for all } 1 \text { A }
$$

In this case, the right-hand side of $(3.60)$ will be negative for all 1fk, which means that all the $P_{1}(t)$ will decrease while the probability $P_{k}$ (associated with the dominant $\beta_{k}$ ) will increase. The significance of thin effect is that the $\beta_{1}$ 's are independent of the residuals and their magnitudes are not determined by which sodel is true. This 1ssue, which has not been discussed in the literature, is believed to tie with the "identifiablility" question of this scheme.

Above discussions merely point out possible shortcomings of this scheme. These lesues may be adequately answered if we could address the following questions.

(1) A rigorous prook to show the asymptotic properties of the hypothesis probabilities. To the best of our knowledge, such a proof is not available in the literature.

(2) How would the hypothesis probabilities behave if none of the models coincide with the true model? Moor and Bawkes (14) used a distance masure to show that the probability associated with the model which 18 the closest one to the true model 
w12l converge to unity. If this claim is warranted, one may be able to design an adaptive paramer alseretisation schere whioh ro-diccretises the paraneter veotor within the parameter abspace wich is the closent to the true model as dotermined by the hypothends probability and the distance measure.

(3) Anowere to the above questions w111 certainly ahade light to the ldentiflability problem.

Flnally, let ue re-emphasize the eignificance of this scheme from the estimation's polnt of view. Th1s algorithm is optiman in the minimum variance sense in state and parameter estimation 1 f the discretized parameter space indeed contains the true parameter. This is true because: (1) we use the conditional man as the estimate and (2) the algorithm was derived without ueing any approximations. 


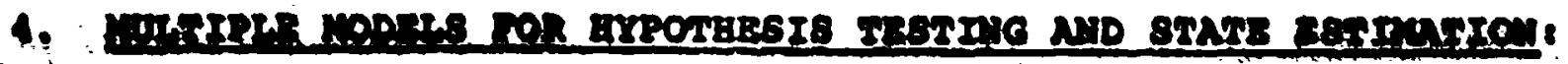

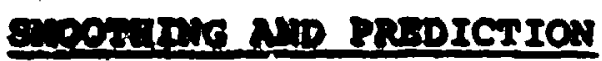

\subsection{Introdupton}

In the previous seotion, we have derived the multiple model filtering algorith for state estimation when the system dyanice contain uncertain parameters. The paraneter vector is discretised to cover a range of phyolcal values that it way possibly attain. A bank of Kalman filters is built with each matchIng to a parameter vector. The a posteriori probability of a given model being true is used to combine the output of these f11ters. Algorithme for state estimation and parameter identiflcation are derived.

In this section, the multiple modes snoothing and prediction algorithms (MMSA and MAPA) are derived.

\subsection{The MSA and MapA: Assumptions}

The system equations, measurement equations, parameter space, and hypothesis probability assumptions made in the section 3.4 are the same for the MSA/MMPA derivation. We only modify the variables of interest to as follows:

1. The conditional mean of the state

$$
\hat{\underline{x}}(\tau / t) \Delta E\{\underline{x}(\tau) / Z(t)\}
$$

2. The conditional state covariance matrix

$$
\underline{\varepsilon}(\tau / t) \underline{\Delta} \operatorname{cov}[\underline{x}(\tau) ; \underline{x}(\tau) / z(t)]
$$


3. The drmanile evolution of the posterior probabilitica $P_{1}(t)$ i egaln, we would like a recureive relation.

(1) when $\tau>t$, it 1s called prodiction. when $T<t$, it is called amoothing. when tot, it is called f1ltering and thle part of algorithm has already been presented.

(2) The conditional moan and the covariance can be computed once the conditional density function has been apecified.

In the following, we restate various forms of prediction and moothing in terme of the evolution of $\mathrm{p}(\underline{x}(\tau) / z(t))$.

(1) Fix lag prodiction/amoothing: update $p(\underline{x}(\tau) / z(t))$ from $p(\underline{x}(\tau-1) / z(t-1))$ where $\tau-t$ is a fixed constant

(2) Fix interval prodiction/smoothing: update $p(\underline{x}(\tau) / z(t))$ from $p(\underline{x}(\tau-1) / z(t))$

(3) Fix point smoothing: update $p(\underline{x}(\tau) / z(t))$ from $P(\underline{x}(\tau) / 2(t-1))$

\subsection{The MSA and MMP: Derivations}

similarly, we start by evaluating the condstional probability deneity function

$$
p(\underline{x}(\tau) / z(t))
$$

Using the marginal density yields

$$
\begin{aligned}
p(\underline{x}(\tau) / Z(t)) & =\int p(\underline{x}(\tau), H / Z(t)) d H \\
& =\int p(\underline{x}(\tau) / H, Z(t)) p(H / Z(t)) d H
\end{aligned}
$$


Bowerer

$$
P(H / z(t))=\sum_{1=1}^{N} P_{1}(t) \delta\left(H-H_{1}\right)
$$

and

$$
P_{1}(t)=\operatorname{Prob}\left(\mathrm{B}-\mathrm{H}_{1} / 2(t)\right)
$$

Notice that $P_{1}(t)$ is interpreted as the probability of the event, $H=H_{1}$, being true conditioned upon all the measurements, $z(t)$. Unlike the state and the covariance $((4.1)$ and $(4.2))$. The hypothes 18 probability ie only a function of one time variable, 1.e." the time index of the measurement space. Using (3.21) and (3.13) In (4.4) yields

$$
p(\underline{x}(\tau) / z(t))=\sum_{i=1}^{N} P_{i}(t) \underline{p}\left(\underline{x}(\tau) / B_{i}, z(t)\right)
$$

This equation is analogous to equation (3.22). Using (4.5), we obtain the predicted/smoothed state and covariance as

$$
\begin{aligned}
\underline{\hat{x}}: \tau / t) & =E\{\underline{x}(\tau) / Z(t)\} \\
& =\int \underline{x}(\tau) p(\underline{x}(\tau) / Z(t)) d \underline{x}(\tau) \\
& =\sum_{i=1}^{N} P_{i}(t) \underline{\hat{x}}_{i}(\tau / t) \\
\underline{E}(\tau / t) & =\operatorname{cov}[\underline{x}(\tau) ; \underline{x}(\tau) / Z(t)] \\
& \left.=\sum_{i=l}^{N} P_{i}(t) \int(\underline{x} / \tau)-\underline{\hat{x}}(\tau / t)\right)(\underline{x}(\tau)-\underline{\hat{x}}(\tau / t))^{T}
\end{aligned}
$$




$$
\begin{gathered}
v p\left(\underline{\underline{x}}(\tau) / B_{1} \cdot s(t)\right) d \underline{x}(\tau) \\
=\sum_{I=1}^{N} P_{1}(t)\left[\underline{\underline{\Sigma}}_{1}(\tau / t)+\left(\underline{\hat{x}}_{1}(\tau / t)-\underline{\hat{\underline{x}}}(\tau / t)\right)\right. \\
\left.\cdot\left(\underline{\hat{x}}_{1}(\tau / t)-\underline{\hat{\underline{x}}}(\tau / t)\right)^{2}\right]
\end{gathered}
$$

where $\hat{\underline{x}}_{1}(\tau / t)$ is the estimate Erom the 1-th spoother/predictor and $\underline{\varepsilon}_{1}(\tau / t)$ is the covariance of $\hat{\underline{x}}_{1}(\tau / t)$.

Ramarks: (1) The realization of MusA/MopA again roquires a bank of amoother/predictor with each matching to a possible parameter vector. The algorithms for the individual smoother/predictor realigation have long been made avallable, for example, see $[3,24-28]$, or Appendix $B$.

(2) Fram the above derivation, the hypothenis probab11itles $P_{i}(t)$ for smoothing/prediction are the same as

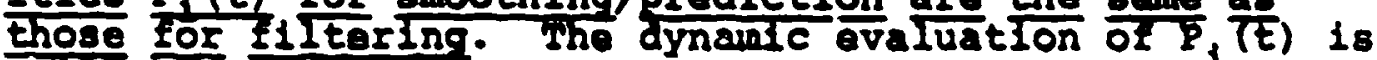
otill computed by using equation (3.47). Recallihg that $P_{1}(t)$ is recursively updated by using the filter residuals. Since the filtering results at time $t$ are $a b-$ tained prior to any prediction and smoothing based upon $z(t)$, the probabilities $P_{1}(t), 1=1, \ldots \ldots N$ are always avallable.

(3) From equations (3.52) and (3.53), the parameter estimate is obtained as the waighted average of discretized parameter vectors. Again, there is only one time Index which is the index of the measurement space. The moothing/prediction algorithr for the parameter est1mate is therefore the same as the filtering algorithm.

In sumary, we state the following procedure for applying MMSA/MMPA.

(1) Compute filtering results, 1.e., obtain $\underline{\hat{x}}_{1}(t / t)$, $\underline{\Sigma}_{i}(t / t), P_{1}(t), \underline{\hat{x}}(t / t)$, and $\underline{\Sigma}(t / t)$ from the algorithms of the previous section.

(2.a) For prediction, apply the individual predictor to 
abtain $\hat{\underline{x}}_{1}(t+k / t)$ and $\underline{\varepsilon}_{1}(t+k / t), 1.0 .1$ 1terate

$$
\underline{\hat{x}}_{1}(t+1 / t)=\underline{A}_{1} \hat{\underline{x}}(t / t)+B_{1} \underline{u}(t)
$$

and

$$
\underline{\Sigma}_{1}(t+1 / t)=\underline{A}_{1} \underline{\Sigma}_{i}(t / t) \underline{A}_{1}^{T}+\underline{L}_{i} \equiv(t) \underline{L}_{1}^{T}
$$

k times with $\underline{\hat{x}}_{i}(t / t)$ and $\underline{\Sigma}_{i}(t / t)$ as initial conditions where $k$ defines the discrete prediction time. The combined estimate $\underline{\hat{x}}(t+k / t)$ and covariance $\underline{\Sigma}(t+k / t)$ are obtained by using $(4.6)$ and (4.7) with the hypothesis probabilities $P_{i}(t)$ the same as those abtained in stap (1) (Elitering).

(2.b) For smoothing, apply the individual smoother (see references [24-28] or Appendix B) to obtain $\hat{x}_{i}(t-k / t)$ and $\underline{\varepsilon}_{i}(t-k / t)$. The combined estimate $\underline{\hat{x}}(t-k / t)$ and covariance $\underline{\Sigma}(t-k / t)$ are obtained by using (4.6) and (4.7) while the hypothesis probabilities $\underline{P_{1}(t)}$ are constant for all $\underline{k}$ and equal to those obtained in step (1). 
5. MULTIPLE MODEL ESTIMATION ALGORITHM EOR NONLINEAR SYSTEMS

In this section, the MEA for nonlinear systems is discused. Fram the pravious section, it is known that the amoothing and prediction are rather etralghtforward extensions, of flltering, only the filtering algorithm will be emphasized here.

similar to the linear case, wo define the following nonInnear system and measurement equations corresponding to the 1 -th discretized parameter vector, $Y_{1}$.

State Dynamics

$$
\underline{x}(t+1)=\underline{f}\left(\underline{x}(t), \underline{u}(t), \underline{\xi}(t), \underline{Y}_{1}\right)
$$

Measurement Equation

$$
\underline{z}(t)=\underline{h}\left(\underline{X}(t), \underline{\theta}(t), \underline{Y}_{1}\right)
$$

The plant noise $\xi(t)$ is defined by equations $(2.4)-(2.7)$ and the measurement noise $\theta(t)$ is defined by equations (2.8) - (2.11). The same as in the linear case, there are three separate steps in the multiple model estimation procedure, namely, the generation of individual state estimates matching to a given parameter vector, the evolution of the hypothesis probability and the combination of the individual estimates. Let each steps be discussed individually below.

(1) It is well-known that the realization of the optimum 
state estimation for systems modelled by (5.1) and (5.2) Involves solving a set of countably infinite differential equations 118 22]. It is therefore practically impossible to obtain these individual optimum estimates. Suboptimum filters will have to be used to construct the filter bank, $1 . e .$, to produce $\hat{x}_{1}(t / t)$ approximately.

(2) The equation for updating the hypothesis probabilIty is stated in equation (3.39)

$$
P_{i}(t+1)=\frac{p\left(\underline{z}(t+1) / H_{i}, z(t)\right)}{\sum_{j=1}^{N} P_{j}(t) p\left(\underline{z}(t+1) / H_{j}, Z(t)\right)} P_{i}(t)
$$

In arriving at this equation, no assumption was made on which type (linear or nonlinear) of systems are being considered. It is therefore still valid for nonlinear estimation. It however, cannot be calculated exactly due to the fact that the exact realization of the individual a posterior density $\mathrm{p}\left(\underline{z}(t+1) / \mathrm{H}_{1}, z(t)\right)$ can not be obtained. It can only be evaluated approximated with a sub-optimal filter (such as the extended Kalman filter*) for computing $\hat{\underline{x}}_{1}(t / t)$ and $\underline{E}_{i}(t / t)$.

(3) Assuming that the optimum individual es:imate $\hat{X}_{i}(t / t)$ and $i t s$ covariance $\Sigma_{i}(t)$ are avallable, the optimum state estimate and its cuvariance can be computed by The extended Kalman filter equations are listed in the Appendix $A$. 


$$
\underline{\hat{x}}(t / t)=\sum_{i=1}^{N} P_{i}(t) \hat{\underline{x}}_{i}(t / t)
$$

and

$$
\begin{gathered}
\underline{\Sigma}(t / t)=\sum_{i=1}^{N} P_{i}(t)\left[\underline{\Sigma}_{1}(t / t)+\left(\underline{\hat{x}}_{1}(t / t)-\underline{\hat{x}}(t / t)\right)\right. \\
\left.\cdot\left(\underline{\hat{x}}_{i}(t / t)-\underline{\hat{x}}(t / t)\right)^{T}\right)
\end{gathered}
$$

Similarly, in order to realize (3.28) and (3.30) for states and measurements represented by (5.1) and (5.2), one has to use suboptimum filters to generate the individual estimates $\underline{\hat{x}}_{i}(t / t)$ and $\underline{\varepsilon}_{i}(t / t)$.

Let us re-emphasize that equations $(3.28),(3.29)$, and (3.30) are exact representations for the solution of the nonlinear estimation problem for systems modeled as (5.1) and (5.2). In other words, the a posterior hypothesis probabilities evolution and the method of computing the combined estimate are optimum if each individual estimate can be obtained optimally.

Numerous siboptimum filters have been proposed for nonlinear estimation $(2,4,28-33\}$. The most popular fjiters are the extended Kalman filter and the second order filter $[2,4]$ among others. Especially, the extended Kalman filter has attracted considerable attentions for practical applications (2-8). The second order filter can generalily provide improved performance 
than the ext " $R^{-}$man filter with the trade-offs of higher computational surden. A comparison of varlous nonlinear filters may be found in $(34,35]$. All these filters may be used for the MABA realization. A specific selection may be based on a particular physical problem and the required performance. For real-time application, one usually favors a simple filter pending on the available computer resources. For off-linear processing especially in the post-mission smoothing application, a sophisticated algorithm is usually preferred. 


\section{ENMPLE}

In this section, we present an example to illuetrate the theory. Only the flitering algorlthm is tested.

Consider the following seoond order continuous syetem.

$$
\left[\begin{array}{l}
\dot{x}_{1} \\
x_{2}
\end{array}\right]=\left[\begin{array}{ll}
0 & 1 \\
0 & -r
\end{array}\right]\left[\begin{array}{l}
x_{1} \\
x_{2}
\end{array}\right]+\left[\begin{array}{l}
0 \\
1
\end{array}\right] u
$$

This aystem may be used to describe the motion of a vehicle along a given axis with drag (represented by "$Y$ ") and control force (represented by " $u$ "). If $x_{1}$ denotes the target range and a radar Is used to take range measurements, the measurement equation 18

$$
2=x_{1}+n
$$

where $n$ is measurement nolse. The measurements are taken at discrete instance of times. A corresponding discrete sysuem of (6..1) 18

$$
\left[\begin{array}{l}
x_{1}(k+1) \\
x_{2}(k+1)
\end{array}\right]=\left[\begin{array}{ll}
1 & \frac{1-e^{-\gamma \Delta t}}{\gamma} \\
0 & e^{-\gamma \Delta t}
\end{array}\right]\left[\begin{array}{c}
x_{1}(k) \\
x_{2}(k)
\end{array}\right]+\left[\begin{array}{c}
0 \\
\frac{1-e^{-\gamma \Delta t}}{\gamma}
\end{array}\right] u
$$

where $\Delta t$ is the time interval between measurements. A multiple model filter is used to estimate $x_{1}, x_{2}$ and to identify $\gamma$. Three $\gamma$ values are assumed, $1 . e ., \gamma=0 ., .5$, or 1.. The system and control matrices, $A$ and $\underline{L}$ for those $\gamma$ values with sampling interval 
$\Delta t=.1$ are

(1) $r=0$.

$$
\underline{\Lambda}=\left[\begin{array}{ll}
1 . & .1 \\
0 . & 1 .
\end{array}\right], \underline{\underline{\underline{y}}}=\left[\begin{array}{l}
0 . \\
.1
\end{array}\right]
$$

(2) $\gamma=.5$

$$
\underline{A}=\left[\begin{array}{ll}
1 . & .0975 \\
0 . & .951
\end{array}\right], \quad \underline{L}=\left[\begin{array}{l}
0 . \\
.0975
\end{array}\right]
$$

(3) $Y=1$

$$
\underline{A}=\left[\begin{array}{cc}
1 . & .095 \\
0 . & .9
\end{array}\right], \underline{\underline{L}}=\left[\begin{array}{l}
0 . \\
.095
\end{array}\right]
$$

The measurement nolse standard deviation is equal to ons. The time initial state is

$$
x_{1}=100, \quad, \quad x_{2}=50 .
$$

The following convention is used to relate the hypothesis to the parameter values.

$$
\begin{aligned}
& \mathrm{H}_{1} \rightarrow \gamma=0 . \\
& \mathrm{H}_{2} \rightarrow \gamma=.5 \\
& \mathrm{H}_{3} \rightarrow \gamma=1 .
\end{aligned}
$$


Two experimonts are parformad. They are deseribed individually below.

Experdmat Is paramter $Y$ stays constant, control i is oqual to sero.

Three cases with the true parameter being equal to one of the three possible values in each case are tested. The a posteriorl hypothesis probabllities for all three cases are plotted in Figure 6.1. The Initial hypothesis probabilities are uniformly distributed. The true system is always identified in within 10 data points

Experiment 2: Parameter $\gamma$ fumps between models, control u is nonzero.

The control force 18 assumed to be equal to 50 and known to the estimator. Assuming the Initial time is zero, the true $Y$ time history is

$$
\begin{array}{lll}
r=0 . & \text { for } & 0 \leq t<2 \\
r=.5 & \text { for } & 2 \leq t<4 \\
r=1 . & \text { for } & 4 \leq t<6
\end{array}
$$

It therefore represents a $\gamma$ history with sudden fumps. The $\gamma$ estimates are presented in Figuze 6.2. Notice that the filter 18 always able to identify the true system. Two modifications are implemented in the algorithm in this case.

(1) The hypothesis probabilities are hard bounded. This is to prevent any probabilities from converging to zero lor 
one). When it doas, it will be very diffleult for the probabillties to branch out again when the true systen has actually owitchad. The bound used in this experiment is however, very anil, 1.e..

$$
\operatorname{Pr}\left[\theta_{1} / 2(t)\right] \geq .0005 \text { for } 1=1,2,3
$$

(2) Although there is no process noise assumed in the system, a process nolse term with covariance

$$
\left[\begin{array}{ll}
1 & 0 \\
0 & 1
\end{array}\right]
$$

is used in the eilters. This is included also for the purpose of preventing the filter from being over-confident in its est1mates therefore not able to switch to a different system. If there 18 no procese noise added, the estimates of a mis-matched filter can drift far away from the true states. When the true parameter jumps to a different value, i.e., an originaliy mismatched filter now becomes matched, it takes extremely long perlod of time for the algorithm to identify the true syatem again. Leaving proper process nolse level in the filter will keep the mis-matched filter estimates sufficiently close to the true state so that the algorithra is adaptive to the parameter jumps. The control variable u also plays a critical role in this experiment. It represents a persistent excitation to explore differences among these systems. A basic issue which still needs answer is on the input design for system identification in using MeA 

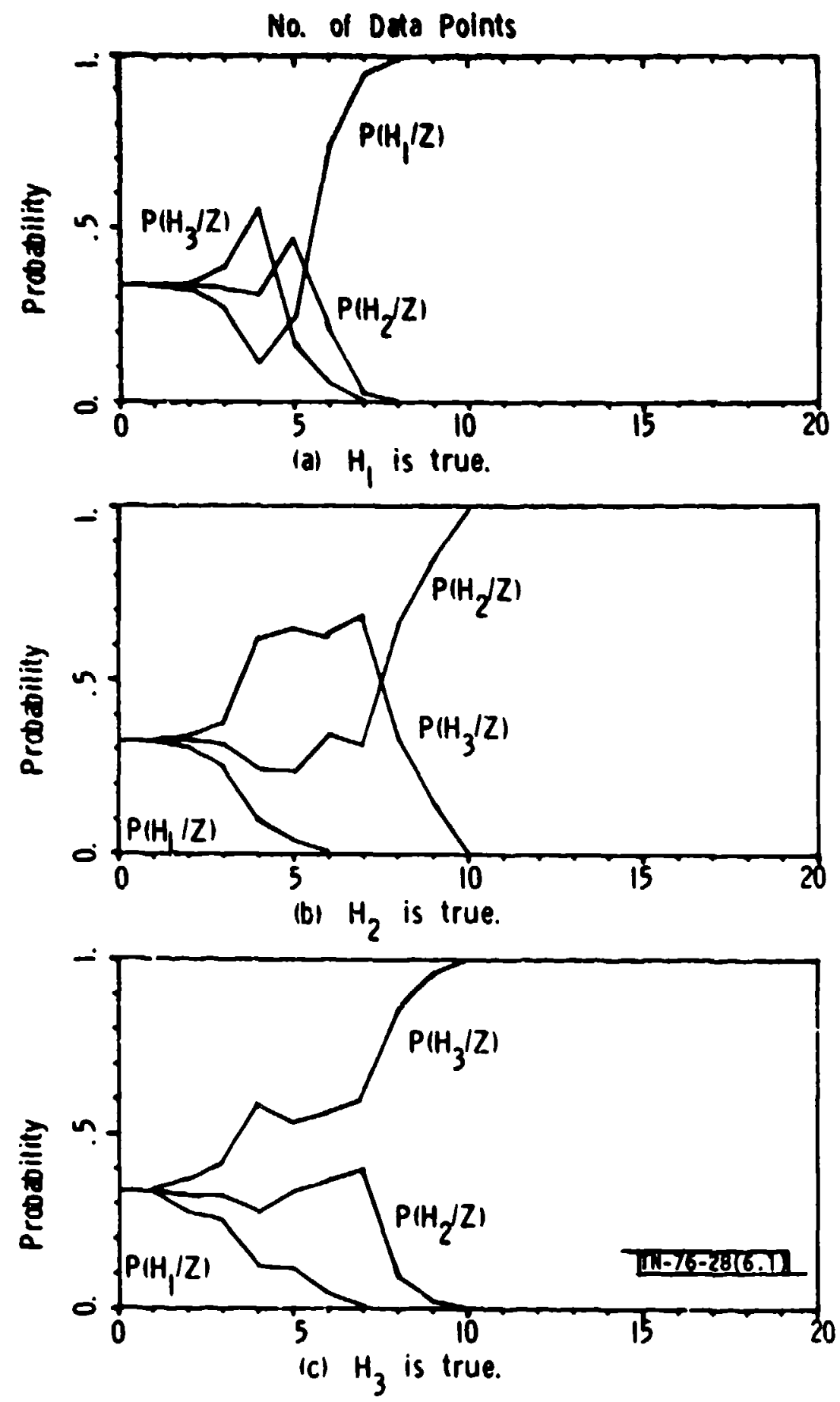

Fig. 6.1 Hypothesis probabilities of experiment 1 


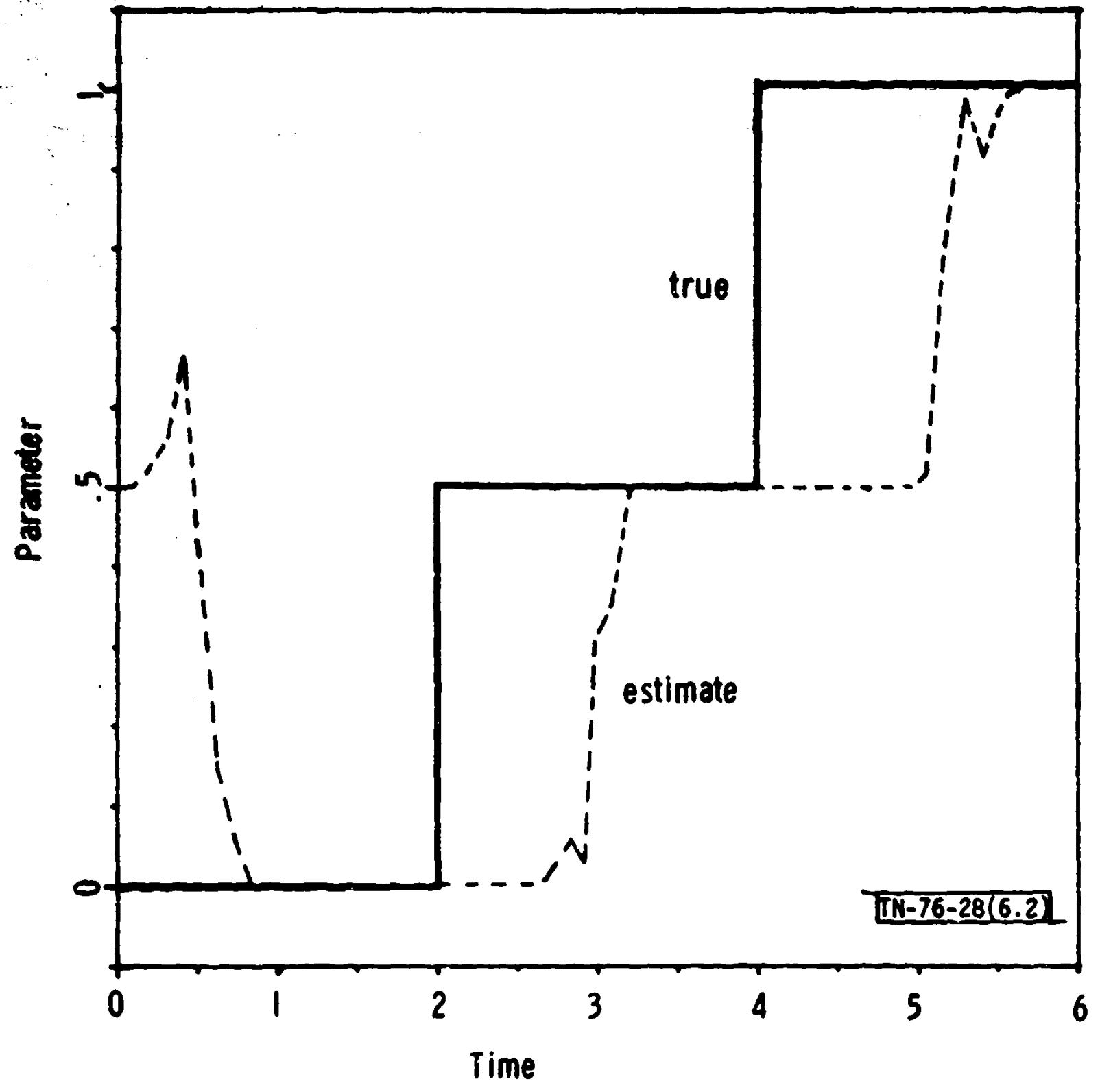

F1g. 6.2 Paxametex estimates of experiment 2 
menod.

The above two experimente are oluple but 1lluetrative.

The firat experiment indicate thet the war can quidoly Identify the true ayoten with a conetant parameter. For time-varying parametera, som modiflcations are neceseary so that the algorithm 1o adaptive to sudden parameter changes. 
7. GUMMARY, DIBCU88ION, ND FURTHER PROBLEMS

7.1 sumary

In this report, we have discuseed the problem of etate estimation with uncertain parameters and presented the colution by utilisating the multiple model entimation algorithm (Mas). The following sumaries pertaining to the properties of MUEA are lieted without any specific order.

(1) Theoretically, the MMEA provides the minimum variance estimates of both state and parameter if one of the chosen models coincides with the true model.

(2) If the a posteriori hypothesis testing probabilities converge asymptotically, the true parameter is identified with probability one.

(3) The hypothesis probabilities for smoothing and prediction are the same as those for filtering.

(4) The hypothesis probability update equation and the weighted sum equations are optimum in the minimum variance sense and they are the same for both $11 \mathrm{n}$ ear and nonlinear systems.

The usefulness of MMEA can only be fully understood and evaluated after applications to significant physical problems. Applications to the trajectory estimation area have still to be carried out. The application to the F-8C airplane real-time control system [16] has shown encouraging results and suggested further study areas in theory as well as in algorithm design. 


\subsection{Discuseton: sxtension to a Clase of Tine-Varying Para- ptere and 8uboptimal Approsebes}

strictly spoaking, the Mus presented in this report is optinu only for ayoten with time-Invarient parameters. The theoretical and practical laplications of using wask to syatems with time-varying parameters are not completely understood. The example in the previous section has clearly indicated that some modifications must be incorporated in order to make the MMEA to follow parameter jump. This is because once the true parameter is ldentified, the algorithm is locked an the true aystem and the mis-matched kalman filter begins to drift away from the true state. When the true parameter has switched to a different value, it usually takes a long time for the algorithm to branch out again. The requirement for a time-varying parameter MEA is to make the mis-matched output still auficiently close to the true state and to keep the hypothesis probability from coming too close to zaro (or unity).

There 1s a triplal extension of the mat to a special clase of time-varying parameters. Consider the parameter space $R_{q}$ which contains $N$ parameter vectors each with dimension $q, 1 . e .$,

$$
R_{q}=\left\{Y: Y=Y_{i} ; i=1, \ldots, N\right\}
$$

At the time $t$, the true parameter is equal to $Y_{1}$. At the next instance of time, the true parameter may be equal to any parameters in $R_{q}$. As time progresses, the true parameter is changing 
around with its values within $R_{q}$. Defining two types of hypothexes by

$H_{1}(t)=$ the hypothesis that $Y$ - $Y_{\text {f }}$ at time $=t$ is true, it

$\bar{B}_{k}(t)=$ the hypothesis that a giving history for time up to $t$ of $y$ indexed by $k$ is true, it is therefore a global hypothesis.

These two types of hypothesis are related by the following equations

$$
\overline{\mathbf{H}}_{k}(t)=\mathrm{H}_{\mathrm{K}_{t}}(t) \bullet \mathrm{H}_{k_{t-1}}(t-1) \bullet \cdots \cdots \mathrm{H}_{k_{1}}(1)
$$

where the index for $k_{1}, \ldots, k_{t}$ is $1, \ldots, N$, the index for $k$ is $1, \ldots \ldots, \mathrm{N}^{t}$, and denotes the "and" operator. It is clear that each $\bar{H}_{k}(t)$ defines a possible sequence of $Y$ history. With this definition, one may proceed in parallel to the development of this report to obtain a new MMEA for time-varying parameters. The derivation is briefly stated below.

1) For state estimate and covariance

Let

$$
P_{i}(t)=\operatorname{Prob}\left(\bar{H}(t)=\bar{H}_{1}(t) / Z(t)\right)
$$

for $1=1, \ldots, N^{t}$. It is trivial to show that

$$
\underline{\hat{x}}(t / t) \cdot E(\underline{x}(t) / 2(t))
$$




$$
\begin{aligned}
& =\sum_{i=1}^{N} P_{1}(t) \underline{\hat{x}}_{1}(t / t)
\end{aligned}
$$

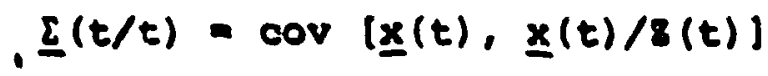

$$
\begin{aligned}
& -\sum_{i=1}^{N} P_{i}(t)\left[\underline{\Sigma}_{1}(t / t)+\left(\hat{\underline{x}}_{1}(t / t)-\underline{\hat{x}}(t / t)\right]\right. \\
& \left.\cdot\left(\underline{\hat{x}}_{1}(t / t)-\underline{\hat{x}}(t / t)\right)^{T}\right) \\
& \underline{\hat{x}}_{i}(t / t)=E\left(\underline{x}(t) / \bar{H}_{i}(t), 2(t)\right) \\
& \underline{\Sigma}_{i}(t / t)=\operatorname{cov}\left[\underline{x}(t), \underline{x}(t) / \bar{H}_{1}(t), 2(t)\right]
\end{aligned}
$$$$
\text { where }
$$

2) For probability update Using the conditional probability relation yields

$$
p(\bar{B}(t+1) / 2(t+1))=p(H(t+1) / \bar{B}(t), 2(t+1)) p(\bar{B}(t) / 2(t+1))
$$

Using Bay's rule on $p(H(t+1) / \bar{H}(t), 2(t+1))$ yields

$$
\begin{aligned}
& P(H(t+1) / \bar{H}(t), Z(t+1)) \\
= & \frac{P(\underline{z}(t+1) / H(t+1), \bar{H}(t), Z(t))}{P(\underline{z}(t+1) / \bar{H}(t), Z(t))} p(H(t+1) / \bar{H}(t), Z(t))
\end{aligned}
$$

Define the following probability density functions 
$P(H(t+1) / \bar{H}(t), 2(t+1))=\sum_{i=1}^{N} P\left(H_{1}(t+1) / \bar{H}(t), 2(t+1)\right) \delta\left(H-H_{1}\right)(7,6)$

$P(H(t+1) / \bar{H}(t), Z(t))=\sum_{i=1}^{N} P\left(H_{i}(t+1) / \bar{H}(t), z(t)\right) \delta\left(H-H_{i}\right)$

where $\left.P\left(H_{i} i t+1\right) / \bar{H}(t), Z(t+1)\right)=\operatorname{Prob}\left(H(t+1)=H_{i}(t+1) / \bar{H}(t), z(t+1)\right)$ and $P\left(H_{j}(t+1) / \bar{H}(t), Z(t)\right)$ is the probability that the parameter will switch to $Y_{i}$ given a past history of $Y$ and all the past measurements. It is determined by the property of the hypothesis process. If the hypothesis process is a Markov process, this probability becomes the transition probability, i.e..

$$
\begin{aligned}
P\left(H_{i}(t+1) / \bar{H}(t), Z(t)\right) & =P\left(H_{i}(t+1) / \bar{H}(t)\right) \\
& =P\left(H_{i}(t+1) / H(t)\right)
\end{aligned}
$$

For example, if the parameter may change to any parameter in $R_{q}$ with equal probability, we may assume

$$
P\left(H_{i}(t+1) / \bar{H}(t), Z(t)\right)=\frac{1}{N} \quad \text { for } i=1, \ldots, N \text {. }
$$

Using (7.6) and (7.7) in (7.5) yields 


$$
\begin{aligned}
& P\left(H_{1}(t+1) / \bar{H}(t), Z(t+1)\right) \\
& =\frac{P\left(\underline{z}(t+1) / H_{1}(\tau+1), \bar{H}(t), Z(t)\right)}{P(\underline{z}(t+1) / \bar{H}(t), Z(t))} P\left(B_{1}(t+1) / \bar{H}(t), Z(t)\right)
\end{aligned}
$$

Using the equation

$$
p(\bar{H}(t) / Z(t))=\sum_{k=1}^{N} P_{k}(t) \&\left(\bar{H}-\bar{H}_{k}\right)
$$

in (7.9) yields

$$
\begin{aligned}
& P\left(H_{i}(t+1) / \bar{H}_{k}(t), z(t+1)\right) \\
& =\frac{P\left(\underline{z}(t+1) / H_{i}(t+1), \bar{H}_{k}(t), z(t)\right)}{P\left(\underline{z}(t+1) / \bar{H}_{k}(t), z(t)\right)} P\left(H_{1}(t+1) / \bar{H}_{k}(t), z(t)\right)
\end{aligned}
$$

where $p\left(\underline{z}(t+1) / H_{1}(t+1), \bar{H}_{k}(t), z(t)\right)$ is the residual density of the filter which was matched to the $k$-th history and is now matched to $Y_{1}$ and

$$
\begin{aligned}
& p\left(\underline{z}(t+1) / \bar{H}_{K}(t), z(t)\right)
\end{aligned}
$$

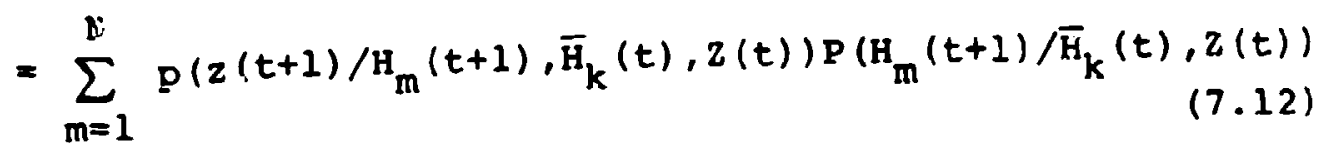$$
\text { Next, we relate } P\left(\bar{H}_{i}(t+1) / Z(t+1)\right) \text { to the conditional }
$$ 
prabability. Uaing

$$
\begin{aligned}
& P\left(B_{1}(t+1) / 2(t+1)\right) \\
& =\int P\left(B_{1}(t+1) / \bar{H}(t), 2(t+1)\right) P(\bar{H}(t) / 2(t+1)) d \bar{H}(t)
\end{aligned}
$$

and equation (7.10) one obtains the following equation for each $\bar{H}_{k}(t)$.

$$
\begin{aligned}
& P\left(H_{i}(t+1), \bar{H}_{k}(t) / 2(t+1)\right) \\
& =P\left(H_{1}(t+1) / \bar{H}_{k}(t), 2(t+1)\right) P\left(\bar{H}_{k}(t) / Z(t+1)\right) \\
& \quad \text { for } 1=1, \ldots, N \text { and } k=1, \ldots, N^{t}
\end{aligned}
$$

where $P\left(H_{1}(t+1) / \bar{H}_{X}(t), Z(t+1)\right)$ is specified in equation $(7.11)$. Notice that $P\left(H_{i}(t+1), \bar{H}_{k}(t) / Z(t+1)\right)$ is the updated hypothesis probabllity. Next, we derive the equation for computing $P\left(\bar{H}_{k} / Z(t+1)\right)$. Using Baye's rule on $P\left(\bar{H}_{k} / Z(t+1)\right)$ yields

$$
\begin{aligned}
& P\left(\bar{H}_{K}(t) / Z(t+1)\right) \\
& =\frac{P\left(\underline{z}(t+1) / \bar{H}_{k}(t), Z(t)\right)}{P(\underline{z}(t+1) / Z(t))} P\left(\bar{H}_{k}(t) / Z(t)\right)
\end{aligned}
$$

where $P\left(\bar{H}_{k}(t) / Z(t)\right)$ is the a posteriori hypothesis probability at time $=t, 1 . e ., P_{k}(t)$. The probability density functions of (7.15) are computed hy using 


$$
\begin{aligned}
& p\left(\underline{z}(t+1) / \mathbb{G}_{k}(t), 8(t)\right) \\
& =\sum_{j=1}^{N} p\left(\underline{g}(t+1) / \bar{R}_{k}(t), H_{j}(t+1), 8(t)\right) P\left(B_{j}(t+1) / \bar{G}_{k}(t), 2(t)\right) \\
& (7,16)
\end{aligned}
$$

and

$$
\begin{aligned}
& P(\underline{z}(t+1) / Z(t)) \\
& =\sum_{m=1}^{N} p\left(\underline{z}(t+1) / \bar{B}_{m}(t), 2(t)\right) P_{m}(t)
\end{aligned}
$$

The probability update is therefore carried out by using equations (7.11), (7.14), and (7.15). These relations can be further condensed with the following simplified notations.

$$
\begin{aligned}
P\left(B_{1} / \bar{B}_{k}, Z(t)\right)=P\left(H_{i}(t+1) / \bar{H}_{k}(t), Z(t)\right) & (7,18) \\
R\left(H_{i} / \bar{B}_{k}\right) & =\frac{P\left(z(t+1) / H_{i}(t+1), \bar{H}_{k}(t), Z(t)\right)}{\sum_{j=1}^{N} P\left(\underline{z}(t+1) / H_{j}(t+1), \bar{H}_{k}(t), Z(t)\right) P\left(H_{j} / \bar{H}_{k}, Z(t)\right)}(7.19) \\
& =\text { conditional likelinood ratio }
\end{aligned}
$$




$$
\begin{aligned}
& f\left(\bar{E}_{k}\right)=\frac{p\left(\underline{E}(t+1) / \bar{E}_{k}(t) \cdot B(t)\right)}{p(\underline{z}(t+1) / 8(t))}
\end{aligned}
$$

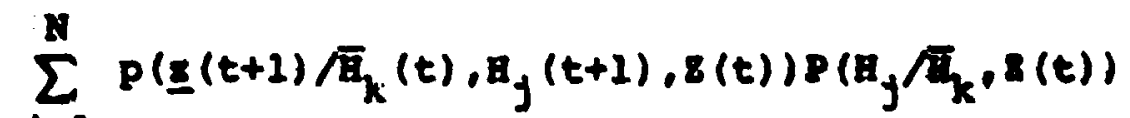

$$
\begin{aligned}
& =\frac{\sum_{1=1}{ }^{t} N}{N} \\
& \sum_{m=1}\left(\sum_{n=1} p\left(\underline{z}(t+1) / \bar{R}_{m}(t), H_{n}(t+1), 8(t)\right) p\left(B_{n} / R_{m}, 8(t)\right)\right) P_{m}(t) \\
& (7.20) \\
& \text { - Iikelihood ratio }
\end{aligned}
$$

Using $(7.18),(7.19)$, and $(7.20)$, equations $(7.11),(7.24)$, and (7.15) may be combined to become

$$
P_{j}(t+1)=l\left(B_{1} / \bar{B}_{k}\right) P\left(B_{1} / \bar{H}_{k}, 2(t)\right) \ell\left(\bar{B}_{k}\right) P_{k}(t)
$$

Notice that for $1=1, \ldots, N$ and $k=1, \ldots, N^{t}$, the indax for $j$ is $1, \ldots . . \mathrm{N}^{t+1}$. The probablilty update is carried out with the conditional probability which characterizes the hypothesis process itself and the likelihood ratios which use the new Information through residual density functions of each filter.

The MEA for a congtant parameter, 1.e., the algorithm discussed in section 3,18 only a degenerate case of (7.21). When the parameter 18 a constant, the local hypotheses, $\mathrm{H}_{1}$, and the global hypotheses, $\overline{\mathrm{H}}_{k}$, become the same. The number of hypotheses 1s limited to the number of parameter vectors in $R_{q}$ Furthermore, the conditional probability of equation (7.11) becomer 


$$
\begin{aligned}
& \dot{p}\left(R_{1}(t+1) / R_{k}(t) .8(t)\right) \\
& \begin{cases}-1 & \operatorname{man} \quad B_{i}=E_{k}=a_{k} \\
-0 & \text { elsewhere }\end{cases}
\end{aligned}
$$

Using $(7.22)$ in $\ell\left(B_{1} / \bar{B}_{k}\right)$ and $\ell\left(\bar{B}_{k}\right)$ yields

$$
\begin{aligned}
& \ell\left(\mathrm{B}_{1} / \overline{\mathrm{B}}_{k}\right)= \begin{cases}1 & \text { when } \mathrm{B}_{1}-\overline{\mathrm{B}}_{k}=\mathrm{g}_{k} \\
0 & \text { elsewhere }\end{cases} \\
& \ell\left(\bar{B}_{k}\right)=\frac{P\left(\underline{z}(t+1) / \mathrm{H}_{k}(t+1), z(t)\right)}{\sum_{m=1}^{N} P\left(\underline{z}(t+1) / H_{m}(t+1), z(t)\right) P_{m}(t)}
\end{aligned}
$$

Using $(7.22),(7.23)$, and $(7.24)$ in $(7.21)$, we obtain equation (3.39), the probability updete equation for the constant parameter case. This completes our a posteriori hypothesis probability derivation.

An obvious problem with this algorithm is that the number $l^{t}$, of $H_{k}(t)$, is growing with $t$. In order to make this algorithm practical, one has to limit the growing number of hypotheses. In the following, several suboptimal approaches for the time-varying parameter MMEA problem are outlined. The first two 
approaches are alnd at liniting the number of poselble sequences (or hypotheces). The last two approaches are mainly to reduce the chance of the algorithm being locked on a particular syetem.

(1) Maximum Iikelihood Probability Approach

Consider the case that at time $t$ there are only $M$ hypotheses selected. For the next time period, each hypothesis may grow with $N$ possibilities. It therefore has M N hypotheses after each filter update. These M - N hypotheses are then limited by selecting only those $M$ which have the largest hypothesis probabilities. (2) Transition Probability and Finite Memory Hypothesis Procese Approach

suppose that the filtering process has limited memory so that $\bar{G}_{k}(t)$ is replaced by the most recent local hypothesis $\mathrm{B}_{k}(t)$. Furthermore, it is assumed that the hypothesis process is a Markov process. Then one is interested in updating

$P\left(B_{1}(t+1) / 2(t+1)\right) \quad ; i=1, \ldots . N$.

from

$P\left(H_{1}(t) / Z(t)\right) \quad$ for all $k=1, \ldots, N$.

With this assumption and using (7.13), one obtains 
$P\left(B_{1}(t+1) / 8(t+1)\right)=\sum_{k=1}^{n} P\left(B_{1}(t+1) / B_{k}(t), 8(t+1)\right)$

- $P\left(R_{k}(t) / 8(t+1)\right)$

(7.25)

where $P\left(B_{1}(t+1) / B_{k}(t), z(t+1)\right)$ is obtained by an equation aimilax to $(7.11), 1.0$.

$P\left(B_{1}(t+1) / B_{k}(t), 8(t+1)\right)$

$=\frac{P\left(\underline{g}(t+1) / B_{1}(t+1), B_{k}(t), z(t)\right)}{P\left(\underline{z}(t+1) / B_{k}(t), z(t)\right)} P\left(B_{1}(t+1) / H_{k}(t), z(t)\right)$

$P\left(q_{k} / 2(t+1)\right)$ is obtained by an equation similar to (7.15), 1.e..

$P\left(H_{k} / Z(t+1)\right)=\frac{P\left(\underline{z}(t+1) / H_{k}(t), Z(t)\right)}{P(\underline{z}(t+1) / Z(t))} P\left(H_{k}(t) / Z(t)\right)$

where

$p\left(\underline{z}(t+1) / H_{k}(t), z(t)\right)$

$=\sum_{j=1}^{R} P\left(\underline{z}(t+1) / H_{k}(t), H_{j}(t+1), Z(t)\right) P\left(H_{j}(t+1) / H_{k}(t), Z(t)\right)$ 


$$
p(g(t+1) / f(t))=\sum_{m=1}^{M} p\left(\underline{g}(t+1) / M_{m}(t), 8(t)\right) P_{m}(t)
$$

Selng $(7.25),(7.26)$, and $(7.27)$ one obtaine en gquation andiar to $(7.21), 1.0 .$,

$P_{j}(t+1)=P\left(B_{j}(t+1) / 8(t+1)\right)$

$=\sum_{k=1}^{N} l\left(B_{j} / B_{k}\right) P\left(B_{j} / B_{k}\right) l\left(B_{k}\right) P_{k}(t)$

where $P\left(B_{j} / B_{k}\right)=P\left(H_{j}(t+2) / H_{k}(t), 2(t)\right)$

- transition probability

$\ell\left(B_{j} / B_{k}\right)=\underset{\text { conditional likelihood ratio defined }}{\text { in }(7.26)}$

$\ell\left(H_{k}\right) \quad=$ likelihood ratio defined in $(7.27)$

The difference of (7.21) and (7.30) 18 that with limited memory, we are interested in $P\left(B_{j}(t+1) / 2(t+1)\right)$ and not in $P\left(\bar{B}_{j}(t+1) / 2(t+1)\right)$. This also limits the number of filters to the number of $Y_{1}^{\prime} s$. One critical issue of this approach is the selection of the transition probability $P\left(B_{j} / H_{k}\right)$. In practical problems, it may be selected a priori with engineering intuition and physical reasons. 


\section{(3) Aging Eilter Approach}

When the syotem dynamice are uncertain and chenging with time, the aging filter $[36,37]$ is often used to place exponentially higher weighting to the moxe recent meanurements. Ite extension to the MasA case $(0.9 .$, in the probability computation) is not avallable. Preliminary results are discuseed in $(38)$.

(4) Others

There exist many methods that can be applied to open up the bandwidth of each Kalman filter and to prevent the a posteriori hypothesis probability from locking on zero (or unity). The method used in the previous section, i.e., increase process noise and bound the probability, is indeed just one of them.

A useful study would be to compare the above approaches by applying them to a significant physical problem, such as the Re-entry Vehicle Tracking problem. 


\subsection{Further Problem Areac}

In this subsection, we conclude by suggesting the followIng further problem areas.

(1) From section 3.7. It was found that some Iundamental issues of MHEA pertaining to its convergence and identifiability still require rigorous investigation. (2) It is demonstrated in section 6 that a known input may be required in come situations to help identlfy timevarying parameters. The problem of optimal signal design in using mus for system Identification is still an open 18sue.

(3) Further studies are required to extend MMEA to timevarying parameters. The optimum MMEA for a special class of time-varying parameters and several suboptimal approaches are discussed in section 7.2 . The extension of MLEA to other types of parameter variation is needed. A Comparative study of the suboptimal approaches is an interesting further topic. 


\section{Acknowledgment}

The authore would like to thank Dr. R. P. Dunn for many Erulteul alequesions. 


\section{APRENTIX A \\ THE DISCRETE RNIMN AND BXTENDAD \\ KAINAN FIITER ALGORITHUS}

In this appendix, we state the discrete Ralman filter elgorlthm and lta f1rot order extension (the extended Ralman ellter) to the nonlinear case.

\section{A.1 The Discrote Kalman Filter Algorithm}

Consider the discrete syatem represented by

$$
\underline{x}(t+1)=\underline{A} \underline{x}(t)+\underline{B} \underline{u}(t)+\underline{I} \underline{\xi}(t)
$$

with measurement equation represented by

$$
\underline{\underline{\varepsilon}}(t+1)=\underline{C} \underline{x}(t+1)+\underline{\theta}(t+1)
$$

where $\underline{x}, \underline{u}$, and $\underline{z}$ are state, control, and measurement vectors, reapectively. $\xi(t)$ and $\underline{\theta}(t)$ are white Gaussian noise sequences with zero mean and covariances $\equiv(t)$ and $\underline{\theta}$, respectively. The matrlces, $\underline{A}, \underline{B}, \underline{\underline{ }}$, and $\underline{\mathrm{C}}$ may be time-varying although not explicitly bhown. The discrete kalman filter algorithm is stated below.

\section{Predict Cycle}

$$
\begin{gathered}
\underline{\hat{x}}(t+1 / t)=\underline{A} \hat{\underline{x}}(t / t)+\underline{B} \underline{u}(t) \\
\underline{\Sigma}(t+1 / t)=\underline{\underline{A}} \underline{\Sigma}(t / t) \underline{A}^{T}+\underline{\underline{L}} \equiv(t) \underline{L}^{T}
\end{gathered}
$$




\section{Update Cycle}

$$
\begin{aligned}
& \underline{\hat{x}}(t+1 / t+1)=\underline{\hat{x}}(t+1 / t)+\underline{w}(t+1)(\underline{z}(t+1)-\underline{c} \hat{x}(t+1 / t)) \\
& \underline{W}(t+1)=\underline{\Sigma}(t+1 / t) \underline{c}^{T}\left[\underline{c} \underline{\Sigma}(t+1 / t) \underline{c}^{T}+\underline{\theta}(t+1)\right]^{-1} \\
& \underline{\Sigma}(t+1 / t+1)=[\underline{I}-\underline{w}(t+1) \underline{C}] \underline{\Sigma}(t+1 / t)
\end{aligned}
$$

where

$$
\begin{gathered}
\underline{\hat{x}}(t / t)=E(\underline{x}(t) / z(t)) \\
\underline{\hat{x}}(t+1 / t)=E(\underline{x}(t+1) / z(t)) \\
\underline{\Sigma}(t / t)=\operatorname{cov}(\underline{x}(t) ; \underline{x}(t) / z(t)) \\
\underline{\Sigma}(t+1 / t)=\operatorname{cov}(\underline{x}(t+1), \underline{x}(t+1) / z(t)) \\
z(t)=\text { the set of all past measurements } \\
=\{\underline{u}(0), \underline{u}(1), \ldots, \underline{u}(t-1), \underline{z}(1), \ldots, z(t)\}
\end{gathered}
$$

The initial estimate $\underline{\hat{x}}(0 / 0)$ is assumed to be Gaussian with mean $\underline{x}(0)$ and covariance $\Sigma(0 / 0)$.

\section{A.2 The Discrete Extended Kalman Filter Algorithm}

Consider a nonlinear system represented by

$$
\underline{x}(t+1)=\underline{f}(\underline{x}(t))+\underline{B} u(t) \cdot+\underline{\underline{L}}(t)
$$

with measurement equation represented by 


$$
\underline{z}(t+1)=\underline{h}(\underline{x}(t+1))+\underline{\theta}(t+i)
$$

where all the matrices and vectors are the same as proviously deflned except that $\underline{\underline{f}}($ ) and $\underline{h}($ ) now represent nonlinear bystem and measurement equatior. espectively. The extended kalman filter is derived by expending $\underline{f}()$ and $\underline{h}()$ in using the raylor series expansion up to first order tern. Let

$$
\begin{aligned}
& \underline{F}=\text { Jacobian matrix of } \underline{\underline{f}}(\text { ) } \\
& =\frac{\partial f(\underline{x}(t))}{\partial \underline{x}(t)} \mid \underline{x}(t)=\underline{\hat{x}}(t / t) \\
& \underline{H}=\text { Jacobian matrix of } \underline{h}() \\
& =\frac{\partial \underline{h(x(t+1))}}{\partial \underline{x}(t+1)} \mid \underline{x}(t+1)=\underline{\hat{x}}(t+1 / t)
\end{aligned}
$$

The discrete extended kalman filter algorithm is stated below. Predict Cycle

$$
\begin{aligned}
\underline{\hat{x}}(t+1 / t) & =\underline{E}(\underline{\hat{x}}(t / t))+\underline{B} u(t) \\
\underline{\Sigma}(t+1 / t) & =\underline{F} \underline{\Sigma}(t / t) \underline{E}^{T}+\underline{L} \equiv(t) \underline{L}^{T}
\end{aligned}
$$

Update cyole

$$
\begin{aligned}
\underline{\hat{g}}(t+1 / t+1) & =\underline{\underline{\varepsilon}}(t+1 / t)+\underline{w}(t+1)(\underline{z}(t+1)-\underline{h}(\underline{\hat{x}}(t+1 / t))) \\
p(I+1) & =\underline{\underline{\Sigma}}(t+1 / \tau) \underline{\underline{H}}^{T}\left[\underline{\underline{H}} \underline{\Sigma}(t+1 / t) \underline{H}^{x}+\underline{\theta}(t+1)\right)^{-1}
\end{aligned}
$$




$$
\underline{\Sigma}(t+1 / t+1)=[\underline{I}-\underline{W}(t+1) \underline{\underline{H}}] \underline{\Sigma}(t+1 / t)
$$




\section{APPENDIX B}

\section{DISCRETE LINBAR SMOOTHING ALGORITEMS}

The system and measurement equations are re-stated

below.

$$
\begin{gathered}
\underline{x}(t+1)=\underline{\underline{A}}(t+1, t) \underline{x}(t)+\underline{B} \underline{u}(t)+\underline{\underline{E}}(t) \\
\underline{z}(t+1)=\underline{c}(t+1) \underline{x}(t+1)+\underline{\theta}(t+1)
\end{gathered}
$$

All the definitions and statistical properties defined in the Appendix A still apply. Notice that the time-varying property of $A(t+1, t)$ and $\underline{C}(t+1)$ is now explicitly shown. We still use the following definition for state estimate and covarianse

$$
\begin{gathered}
\underline{\hat{x}}(\tau / t)=E[\underline{x}(\tau) / Z(t)] \\
\underline{\Sigma}(\tau / t)=\operatorname{cov}[\underline{x}(\tau) ; \underline{x}(\tau) / Z(t)]
\end{gathered}
$$

Three kinds of smoothing are considered. They are defined below.

(1) Fixed-interval smoothing: given $z(T)$, obtain $\underline{\hat{x}}(t / T)$ and $\underline{E}(t / T)$ for all $t<T$.

(2) Fixed-point smoothing: given $\tau$, obtain $\underline{\hat{x}}(\tau / t)$ and $\underline{\varepsilon}(\tau / t)$ for all $t>\tau$.

(3) Fixed-lag smoothing: advance $\hat{x}(t / t+k)$ and $\underline{\Sigma}(t / t+k)$ to $\underline{\hat{x}}(t+1 / t+1+k)$ and $\underline{\varepsilon}(t+1 / t+1+k)$ where $k$ is a positive constant. 
Only the algorithms will be stated here. Their derivatien: why be cound in many references, e.g., refs [24-28]. These algorlen are stated individually in the following subsections.

\section{-1 Exad-interval Smoothing Algorithm}

In order to use the fixed-interval smoothing algorithm, the filtering resulta must be first made available.

\section{State}

$$
\underline{\hat{\hat{x}}}(t / T)=\underline{\hat{x}}(t / t)+\underline{G}(t)[\underline{\underline{R}}(t+1 / T)-\underline{g}(t+1 / t)]
$$

Gain

$$
\underline{G}(t)=\underline{\Sigma}(t / t) \underline{A}^{T}(t+1, t) \underline{\Sigma}^{-1}(t+1 / t)
$$

Covarlance

$$
\underline{E}(t / T)=\underline{\Sigma}(t / t)+\underline{G}(t)[\underline{\Sigma}(t+1 / T)-\underline{\Sigma}(t+1 / t)] \underline{G}^{T}(t)
$$

\section{Initial Conditions}

$$
\underline{\hat{R}}(T / T), \underline{\Sigma}(T / T)
$$

\section{B. 2 Fixed-point Smoothing Algorithm}

There are several equivalent algorithms in this category. Only one of them is stated here. Similsry, the filtering results are needed for fixed-point smoothing.

\section{State}

$$
\underline{\hat{x}}(\tau / t)=\hat{x}(\tau / t=1)+\underline{D}(I / t)+\underline{\hat{x}}+t / t)=\underline{\underline{x}}(t / t-1)]
$$




\section{caln}

$$
\underline{D}(\tau / t)=\underline{D}(\tau / t-1) \underline{\Sigma}(t / t) \underline{A}^{T}(t+1, t) \underline{\Sigma}^{-1}(t+1 / t)
$$

Covarlance

$$
\underline{\Sigma}(\tau / t)=\underline{\Sigma}(\tau / t-1)-\underline{D}(\tau / t) \underline{\underline{W}}(t) \underline{C}(t) \underline{\Sigma}(t / t-1) \underline{D}^{T}(\tau / t)
$$

Initial Condition

$$
\underline{\hat{x}}(\tau / \tau), \underline{\Sigma}(\tau / \tau), \underline{D}(\tau / \because)=\underline{I}
$$

where $w(t)=$ filtering gain defined in (A.6).

$$
\underline{I}=1 \text { dentity matrix. }
$$

\section{B.3 Fixed-lag Smoothing Algorithm}

In order to perform fixed-lag smoothing, the filtering, fixed-interval smoothing, and fixed-point smoothing results must be avallable to obtain initial conditions.

\section{State}

$$
\begin{aligned}
& \underline{\hat{x}}(t+1 / t+1+k)=\underline{A}(t+1, t) \hat{\mathbf{x}}(t / t+k)+\underline{B} u(t) \\
& +\underline{\underline{E}} \equiv(t) \underline{\underline{L}}^{T} \underline{A}^{-T}(t+1, t) \underline{\varepsilon}^{-1}(t / t)[\hat{\mathbf{x}}(t / t+k)-\hat{\hat{x}}(t / t)] \\
& +\underline{E}(t+1 / t+1+k) \underline{w}(t+1+k)[\underline{z}(t+1+k)-\underline{c}(t+1+k) \underline{\hat{x}}(t+1+k / t+k)](B .13)
\end{aligned}
$$

Gain

$$
\underline{D}(t+1 / t+1+k)=\underline{G}^{-1}(t) \underline{D}(t / t+k) \underline{G}(t+k)
$$




\section{Covariance}

$$
\begin{aligned}
& \underline{\Sigma}(t+1 / t+1+k)=\underline{\Sigma}(t+1 / t)-\underline{D}(t+1 / t+1+k) \\
& -\underline{\underline{M}}(t+k+k) \underline{c}(t+1+k) \underline{\Sigma}(t+1+k / t) \underline{D}^{T}(t+1 / t+1+k) \\
& -\underline{G}^{-1}(t) \cdot[\underline{\Sigma}(t / t)-\underline{\Sigma}(t / t+k)] \underline{G}^{-T}(t)
\end{aligned}
$$

where $M(t)=$ filtering gain, defined in $(A, \sigma) . G(t)=$ fixed-interval smoothing gain, defined in (B.6).

Initial Conditions

$$
\underline{\hat{x}}\left(t_{0} / t_{0}+k\right), \underline{\Sigma}\left(t_{0} / t_{0}+k\right), \underline{D}\left(t_{0} / t_{0}+k\right)
$$

These conditions are obtained from fixed-point smoothing. 
REFERENCES

11) R. B. Kalman, "A New Approach to Linear Filtering and Prediction Probleme," Trans. Asks, J. Basic Eng., 8er. D, 82. 34-45, (1960).

(2) A. H. Jaswinak1, Stochastlc Proceses and P1Itering Theory (Acadenic Prese, Now York (1970).

(3) A. Gelb, ed. Applied Optimal Betimation (M.I.T. Press, Cambrldge, Massachusetts, (1974).

(4) M. Athans, R. H. Whiting, and M. Gruber, "A Suboptimal Betimation Algorithm with Probabilistic Editing for False Measurements with Applications to Target Tracking with Wake Phenomena," to appear in IBEE Trans. Automatic Control.

[6] C. B. Chang, R. H. Whiting, and M. Athans, "On the state and Parameter Batimation for Maneuvering Re-entry Vehicles," to appear In IEEE Trans. Automatlc Control.

(7) C. B. Chang, R. H. Whiting, and M. Athans, "Application of Adaptive Filtering Methods to Maneuvering Trajectory Estimation," Technical Note 1975-59, Lincoln Laboratory, M.I.T. (24 November 1975), DDC AD-B008137-L.

[8] M. Gruber "An Approach to Target Tracking," Technical Note 1967-8, Lincoln Laboratory, M.I.T. (10 February 1967), DDC AD-654272.

(9) M. Athans and P. P. Varalya, "A Survey of Adaptive stochastic Control Methods," Proc. Engineering Foundation Conference on systems Engineering, New England College, Henniker, N.H., Auguet 1975, also submitted to IEEE Trans. Automatic Control.

[10] D. T. Magill, "Optimal Adaptive Estimation of Sampled Stochastic Processes," IEEE Trans. Automatic Control AC-10, $434-439$ (1965). 
[11] D. G. Lainiotis, "Optimal Adaptive sotimations Structure and Paramoter Adaptation," IEst Trang. Automatic Control AC-15, 160-170 (1971).

[12] D. Willner, "Observation and Control of Partially Unknown Systems," Ph.D. Diesertation, Department of Electrical Engineering, M.I.T., also M.I.T. Electronic Systeme Laboratory Report ESL-R-496 (June 1973).

[13] T. N. Upadhyay and D. G. Lainiotis, "Joint Adaptive Plant and Measurement Control of Iinear Stochastic Systems," IBEB Trans. Automatic Control AC-19, 567-571 (1974).

[14] J. B. Moore and R. M. Hawkes, "Decision Methods in Dynamic System Identification, "Proc. of 1975 IEEE Conf. on Declsion and Control, Houston, Texas, pp. 645-650.

[15] M. Athens and D. Willner, "A Practical Scheme for Adaptive Aircraft Flight Control Systems," Proc. Symposium on Parameter Estimation Techniques and Applications in Aircraft Flight Testing, NASA TN D-7647, NASA Flight Research Center, Edwards, Calif. (April 1973), pp. 315-336.

[16] M. Athans, K. P. Dunn, C. S. Greend, W. H. Lee, N. R. Sandell, Jr., I. Segall, and A. S. willsky, "The Stochastic Control of the F-8C Alrcraft Using the Multiple Model Adaptive Control (xMAC) Method," Proc. of 1975 IzeE Conf. on Decision and Control, Houston, Texas, pp. 217-228.

[17] A. II. Jaswinski, "Adaptive Filtering," Automatica 5, 475485 (1969).

[18] H. J. Kushner, "On the Differential Equations Satisfied by Conditional Probability Densities of Markiv Processes with Applications," J. SIAy Control, Ser. A 2, 106-119 (1964).

[19] H. J. Kushner, "Dynamic Equations for Optimum Nonlinear Filtering," J. Differsr.tial Equations 3, 179-190 (1967). 
[20] H. J. Kushner, "Nonlinear Filtering: the Exact Dynamical Equations Satisfied by the Conditional Mode," IEEE Trans. Automatic Control AC-12, 262-267, (15:7).

[21] R. S. Bucy, "Nonlinear Filtering," IEEE Trans: Automatic Control AC-10, 198 (1965).

[22] M. Wonham, "Some Applications of Stochastic Differential Equations to Optimal Nonlinear Filtering," J. SIAM Control, Ser. A 2 , 347-369 (1965).

[23] A. H. Jazwinski, "Nonlinear Filtering - Numerical Experiments," NASA - Goddard Astrodynamics Conf., Greenbelt, Maryland, April 1966.

[24] J. S. Meditch, Stochastic Optimal Linear Estimation and Control (McGraw-Hill, New York, 1969).

[25] J. S. Meditch, "Optimal Fixed Point Continuous Linear Smoothing," Proc. Joint Automatic Control Conf., June 1967, pp. 249-257.

[26] J. S. Meditch, "On Optimal Linear Smoothing Theory," Information and Control, 10, 598-615 (1967).

[27] J. S. Meditch, "A Successive Approximation Procedure for Nonlinear Data Smoothing," Proc. Symposium on Inform. Processing, Purdue University, Lafayette, Indiana, April 1969, pp. 555-568.

[28] A. P. Sage and J. L. Melsa, Estimation Theory with Applications to Communications and Control (McGraw1971).

[29] B. Friedland and I. Bernstein, "Estimation of the State of a Nonlinear Process in the Presence of Nongaussian Noise and Disturbances," J. Franklin Institute 281, 455-480 (1966).

[30] H. Cox, "On the Estimation of the State Variables and Parameters for Noisy Lynamic Systems," IEEE Trans. Automatic Control AC-9, 5-12 (1964). 
(31) V. O. Nowery. "Ieast Squara Recurelve Differential Correction freination in Nonilnear Probleme," IEBs Trane. Automat1o Control AC-10, 399-407 (1965).

(32) D. Dotchmendy and R. Sridhar, "Seguential betimation of 8tates and Parameters in Noley Nonlinear Dynamical syetems." Trane. ABN. J. Basic Engrg. 362-368 (1966).

(33) R. W. Base, V. D. Norum, and L. Schwartz, "Optimal Multichannel Nonlinear Filtering," J. Math. Analyols and Applications 16, 152-164 (1966).

(34) L. Schwarts and E. B Stear, "A Computational Comparison of Several Nonlinear Filters," IEEE Trana. Automatic Control AC-13, 83-86 (1968).

[35] R. P. Miahner, J. A. Tabaczynski, and M. Athans, "A Comparison of Three Non-Linear Filters," Automatica 5, 487-496 (1969).

(36) T. J. Tarn and J. Zaborszky, "A Practical, Nondiverging Filter, AIAA J. 8, 1127-1133 (1970).

[37] R. W. Miller, "Asymptol- Behavior of the Kalman Filter with Exponential Aging," AIAA J. 9. 537-538 (1971).

[38] K. P. Dunn, "The Multiple Model Eatimation Algorithm with Exponential Aging," to be issued, 